\title{
From wrinkling to global buckling of a ring on a curved substrate
}

\author{
R. Lagrange ${ }^{\mathrm{a}}$, F. López Jiménez ${ }^{\mathrm{b}}$, D. Terwagne ${ }^{\mathrm{b}, 1}$, M. Brojan $^{\mathrm{c}, 2}$, P. M. Reis ${ }^{\mathrm{b}, \mathrm{c}, *}$ \\ ${ }^{a}$ Department of Mathematics, Massachusetts Institute of Technology, Cambridge, MA 02139, USA \\ ${ }^{b}$ Department of Civil and Environmental Engineering, Massachusetts Institute of Technology, Cambridge, MA 02139, USA \\ ${ }^{c}$ Department of Mechanical Engineering, Massachusetts Institute of Technology, Cambridge, MA 02139, USA
}

\begin{abstract}
We present a combined analytical approach and numerical study on the stability of a ring bound to an annular elastic substrate, which contains a circular cavity. The system is loaded by depressurizing the inner cavity. The ring is modeled as an Euler-Bernoulli beam and its equilibrium equations are derived from the mechanical energy which takes into account both stretching and bending contributions. The curvature of the substrate is considered explicitly to model the work done by its reaction force on the ring. We distinguish two different instabilities: periodic wrinkling of the ring or global buckling of the structure. Our model provides an expression for the critical pressure, as well as a phase diagram that rationalizes the transition between instability modes. Towards assessing the role of curvature, we compare our results for the critical stress and the wrinkling wavelength to their planar counterparts. We show that the critical stress is insensitive to the curvature of the substrate, while the wavelength is only affected due to the permissible discrete values of the azimuthal wavenumber imposed by the geometry of the problem. Throughout, we contrast our analytical predictions against finite element simulations.
\end{abstract}

Keywords:

Elasticity, Instability, Buckling, Wrinkling, Ring, Substrate

\section{Introduction}

Wrinkling is a stress-driven mechanical instability that occurs when a stiff and slender surface layer, bonded to a compliant substrate, is subject to compression. This universal instability phenomenon is found in numerous natural and technological/engineering examples, over a wide range of length scales, including: carbon nanotubes (Lourie et al., 1998), pre-stretched elastomers used in flexible electronics applications (Kim et al. 2011), human skin (Chen and Yin, 2010), drying fruit (Yin et al., 2009), surface morphology of the brain (Budday et al. 2014) and mountain topographies generated due to tectonic stresses (Price and Cosgrove, 1990: Huddleston and Lan, 1993).

Over the past decade, there has been an upsurge of interest in the study of the mechanics of wrinkling, along with a change of paradigm in regarding surface instabilities as an opportunity for functionality, instead of a first step in the route to structural failure (Genzer and Groenewold, 2006, Li et al., 2012). The first mechanical studies of wrinkling were motivated by the stability of sandwich panels (Allen, 1969), used in lightweight structural applications, in which the core acts as a soft substrate for the much stiffer skin. More recently, Bowden et al. (1998) showed how the wrinkling of a thin film on an elastomeric substrate can be used to produce complex self-organized patterns. Their seminal work has instigated the realization of

\footnotetext{
${ }^{*}$ Corresponding author at: Department of Mechanical Engineering and Department of Civil \& Environmental Engineering, Massachusetts Institute of Technology, Cambridge, MA 02139, USA. Tel.: +1 6173243325. E-mail address: preis@mit.edu (P.M. Reis).

${ }^{1}$ Current address: Department of Physics, Faculté des Sciences, Université Libre de Bruxelles (ULB), Bruxelles 1050, Belgium.

${ }^{2}$ Current address: Faculty of Mechanical Engineering, University of Ljubljana, Slovenia.
}

Preprint submitted to Journal of the Mechanics and Physics of Solids

May 7, 2015 
wrinkling through several different actuation mechanisms, including thermal mismatch (Huck et al., 2000), tissue growth/atrophy (Ben Amar and Goriely, 2005, Li et al., 2011a; Budday et al., 2014), swelling by a liquid (Chan and Crosby, 2006b) or vapor solvent (Breid and Crosby, 2009), and pneumatics (Terwagne et al. 2014). The opportunities in applications opened by such a wide range of external stimuli have enabled the usage of wrinkling in photonics (Kim et al. 2012), optics (Chan and Crosby) 2006a), self-assembly (Yoo et al. 2002), microfluidics (Yin et al., 2012) and morphogenesis (Efimenko et al., 2005).

In order to provide a theoretical background to these recent developments, several authors have built on the pioneering work of Allen (1969), who first provided close form solutions for the critical stress and wavelength obtained when an initially straight beam, adhered to an infinite plane substrate, is placed under a state of uniaxial compression. Chen and Hutchinson (2004) extended this work to consider the case of a plate adhered to a flat substrate under equi-biaxial compression and performed a nonlinear analysis of the Föppl-von Kármán equations (Landau and Lifshitz, 1959, Timoshenko and Gere, 1961). Huang et al. (2005) further refined these efforts by considering the effect of a finite substrate. Both studies showed the existence of multiple buckling modes associated with the same value of critical stress. The stability of these modes under different loadings conditions has been addressed by Audoly and Boudaoud (2008a b c c), who produced a stability diagram covering the evolution from low to high values of overstress. However, experiments by Cai et al. (2011) found disagreement at low values of overstress, suggesting that a finite intrinsic curvature of their experimental system, even if small, may play an important role in dictating pattern selection.

Early studies of wrinkling on curved substrates, as in the flat configuration, were also motivated by a structural problem; in this case, in the context of the stability of the outer shell of rockets (Kachman, 1959: Seide and Weingarten, 1961, Seide, 1962). More recent studies that consider instabilities as a possible source of functionality have led to applications of curved configurations in adhesion (Kundu et al., 2011), microfluidics (Mei et al., 2010), morphogenesis of microparticles (Yin et al., 2014), optics (Breid and Crosby, 2013) and aerodynamic drag reduction (Terwagne et al., 2014). Curvature also plays a relevant role in the growth of biological systems (Li et al., 2011b). Despite these important emerging applications, the mechanics of wrinkling on curved substrates remains poorly understood, when compared to the planar counterpart.

Systematic Finite Element simulations of wrinkling in curved systems have been performed (Yin et al. 2009: Chen and Yin, 2010, Li et al., 2011c, Cao et al., 2012) that highlighted a complex pattern formation process. These numerical studies also suggested the possibility for curvature to affect the selected patterns and modify the relevant characteristic length scales, which calls for a robust theoretical backing. Analytical predictions are challenged by the difficulty of modeling the stiffness of the substrate, even in two-dimensional configurations. Cheng (1996) and Cai et al. (2011) used the stiffness provided by Allen (1969) for the flat case, such that their model therefore neglects the contribution of curvature on the response of the substrate. Yin et al. (2009) used the prediction provided by Brush and Almroth (1975), which accounts for curvature but does not consider its influence on the wrinkling wavelength and their prediction does not converge to the classical planar case when the curvature tends to zero. As such, there is a need to quantify the effect of curvature on the stiffness of the substrate and its subsequent influence on wrinkling.

Here, to the best of our knowledge, we provide the first analytical work that accounts for both the curvature of a (2D) shell-substrate system, as well as the finite size of the substrate. As an initial step, we focus our study on a curved film adhered to a cylindrical substrate, instead of dealing with non-zero Gaussian curvature geometries, which is left for a future study. We assume axial-symmetry to further simplify the system to the 2D problem of a ring on an annular substrate. Mechanical loading is applied by depressurizing a circular cavity inside the substrate, which places the system under a state of compression. This geometry is motivated by recent experiments (Terwagne et al., 2014) that demonstrated the usage of wrinkling on spherical samples for switchable and tunable aerodynamic drag reduction. In our simplified 2D system, we solve the elasticity problem for the substrate and derive a close form expression for its stiffness, which is then used in the stability analysis of the ring to quantify the buckling patterns.

The paper is organized as follows: In $\S 2$, we introduce our system along with its material and geometrical parameters. We also describe the possible instability modes, and present a simplified phase diagram, with the aim of providing physical insight on the problem. In $\S 3$, we then introduce the kinematics of the ring attached to the substrate and determine the stiffness of the substrate. We proceed by defining a strain energy that includes both bending and stretching of the ring, as well as the effect of the substrate. Energy 
minimization yields the equilibrium equations of the problem. An asymptotic expansion is then used to calculate the principal solution and the bifurcation at the onset of instability. In $\S 4$, we describe the finite element simulations that we have performed for this same system.

The results of our investigation are presented in $\S 5$. Throughout, we directly compare the analytical predictions to the numerical simulations. We start with the fundamental solution and the critical conditions that lead to instability. We then construct a phase diagram which rationalizes the dependence of the instability modes on the governing parameters. The results for our system are then quantitatively compared to those for wrinkling of a film on a planar substrate, highlighting the effect of curvature. Finally, $\S[6$ summarizes our findings and provides perspectives for potential extensions of our work in future studies.

\section{Definition of the problem}

We study the stability of a thin elastic ring, bound to an equally curved 2D substrate that contains an inner cavity, a schematic diagram of which is presented in Fig. 1(a). The system is initially at equilibrium, with identical pressures inside and outside of the sample. Motivated by recent experiments on spherical specimens (Terwagne et al. 2014), the system is then loaded by applying a depressurization, $\mathrm{P}$, to the inner cavity. The thickness of the ring is $H$, its Young's modulus $E_{F}$ and its Poisson's ratio $\nu_{F}$. We refer to $\overline{E_{F}}=E_{F} /\left(1-\nu_{F}^{2}\right)$ as the reduced Young's modulus of the film. The substrate is made of a linearly elastic material with Young's modulus $E_{S}$, Poisson's ratio $\nu_{S}$ and reduced Young's modulus $\overline{E_{S}}=E_{S} /\left(1-\nu_{S}^{2}\right)$. The thickness of the substrate is $R-R_{0}$, where $R_{0}$ is the radius of the inner cavity.

For convenience, we now introduce new rescaled quantities to reduce the number of parameters of the problem. As such, we use $R$ and $\overline{E_{F}}$ to normalize lengths and pressures and define

$$
h=\frac{H}{R}, \beta=\frac{R_{0}}{R}, \quad \xi=\frac{\overline{E_{F}}}{\overline{E_{S}}}, p=\frac{P}{\overline{E_{F}}},
$$

as the dimensionless thickness, cavity size, stiffness ratio, and pressure, respectively.

The principal solution corresponds to an axisymmetric deformation that leads to a decrease of both the inner and outer radii. As the depressurization increases, the onset of instability is reached. In Fig. 1(b-e) we show representative results obtained from Finite Element Modeling (FEM), of the three possible instability configurations of the ring-substrate system, for different values of the dimensionless ring thickness, $h$, and ratio of stiffness, $\xi$. For these results, all the other mechanical properties were kept constant: the Poisson's ratios of the film and substrate are $\nu_{F}=\nu_{S}=0.5$, and the dimensionless size of the cavity is $\beta=0.2$. In the simulations, this is achieved by fixing $R=100$ units of length and $E_{S}=1$ units of pressure, while changing the values of $H, R_{0}$ and $E_{F}$ accordingly. More details of our numerical simulations are provided in $\$ 4$

The first mode, a representative example of which is shown in Figure 1 (b) for $h=10^{-2}$ and $\xi=10^{3}$, corresponds to periodic wrinkling of the film with a well defined wavelength. The displacements are localized in the region close to the film. The second instability mode, for example at $h=10^{-2}$ and $\xi=10^{6}$ in Figure 1 (c), corresponds to a global buckling of the structure, where both the ring and the cavity deform into an ellipse such that the wavelength is $\lambda=\pi R$.

In addition to these two instability modes (wrinkling and global buckling), we have also numerically observed an instability on the inner surface of the cavity. However, this third mode does not affect the ring and is only found for low values of cavity size and stiffness ratio $\left(\xi=10^{2}\right.$ and $h=10^{-2}$ in Fig. 11-d). This instability was first discussed by Biot (1965) and we therefore refer to it as the Biot mode; it is local in nature and only depends on the compressive strain at the inner surface. This type of instability mode has been recently studied in the case of elastomeric materials with voids by Michel et al. (2007) and Cai et al. (2010). Understanding the specifics of this Biot mode is however outside the scope of our work and we shall not take it into account in our analytical model and systematic numerical investigation.

A schematic phase diagram of our system is provided in Fig. 2. For low values of $h$ and $\xi$, the ring wrinkles with a short wavelength. As either $h$ or $\xi$ are increased, the wavelength also increases. Once these parameters reach a critical value, represented by the dashed line in Fig. 2, the instability transitions from wrinkling to global buckling. In what follows, we focus on rationalizing how the wavelength of the wrinkling 
mode, and the threshold value for the transition to global buckling, evolve with the elastic and geometrical parameters of the system.

\section{Analytical model}

The ring is treated as an Euler-Bernoulli beam. The effect of the substrate is modeled as a restoring force that acts on the ring and is determined by solving the elasticity problem of the substrate with adequate boundary conditions. Minimization of the potential energy provides the equilibrium equations of the problem, which are solved using an asymptotic expansion that yields the principal and bifurcated solutions.

\subsection{Kinematics, energy formulation and equations of equilibrium}

We model the ring as an extensible Euler-Bernoulli beam made of an homogeneous and isotropic material. Polar coordinates are used to track the position of the ring center-line, $C$. The initial configuration of the ring, prior to depressurization, is assumed to be circular. The origin, $O$, is located at the center of the cavity, and the initial and equilibrium configurations of an arbitrary point of $C$ are represented by $M_{0}$ and $M$, respectively. Vectors are expressed in the physical base $\left(\mathbf{e}_{\mathbf{r}}, \mathbf{e}_{\theta}\right)$, derived from the polar coordinates $(r, \theta)$. The initial position of $C$ is $\mathbf{O M}_{0}=(R, 0)$, as shown in the inset of Fig.1(a). When the system is loaded by depressurizing the cavity, $C$ deforms into a new configuration given by the position vector $\mathbf{O M}=R(1+v(\theta), u(\theta))$, where $v$ and $u$ are the dimensionless radial and orthoradial displacements, respectively.

The infinitesimal arclength of $C$ in the initial and deformed configurations are denoted by $d s_{0}=\left|d \mathbf{O M}_{0}\right|$ and $d s=|d \mathbf{O M}|$, respectively. Moreover, defining the tangent vector $\mathbf{T}=d \mathbf{O M} / d s$, allows us to express the curvature of $C$ in the deformed configuration as $\kappa / R=|d \mathbf{T} / d s|$. Here, $\kappa$ is dimensionless and can be written in terms of $v$ and $u$ as

$$
\kappa=1+\left(-1+2 u^{\prime}+2 v\right) v^{\prime \prime}+v^{2}-v-u^{2}+\left(-u+v^{\prime}\right) u^{\prime \prime}+\frac{1}{2}\left(v^{\prime 2}-u^{2}\right)+\text { h.o.t. }
$$

where the prime notation represents derivation with respect to $\theta$ and high order terms (h.o.t.) are neglected under the assumption of small displacements and moderate rotations. We now define the elongation of the ring as $e=d s / d s_{0}$ to express the stretching deformation, $\eta=\left(e^{2}-1\right) / 2$, in terms of $v$ and $u$ as

$$
\eta=\frac{1}{2}\left[u^{\prime 2}+v^{2}+\left(u-v^{\prime}\right)^{2}\right]+(1+v) u^{\prime}+v
$$

so that the hoop stress in the film is $\sigma_{0}=\overline{E_{F}} \eta$.

Following Euler-Bernoulli beam theory (Timoshenko and Gere, 1961), the total energy of deformation $\mathcal{E}$ of the ring is the sum of a stretching energy $\mathcal{E}_{S}$ and a bending energy $\mathcal{E}_{B}$,

$$
\mathcal{E}=\mathcal{E}_{S}+\mathcal{E}_{B}=\int_{0}^{2 \pi R} \overline{\mathcal{E}} d s_{0}
$$

with

$$
\begin{aligned}
\mathcal{E}_{S} & =\int_{0}^{2 \pi R} \frac{\overline{E_{F}} H}{2} \eta^{2} d s_{0} \\
\mathcal{E}_{B} & =\int_{0}^{2 \pi R} \frac{\overline{E_{F}} H^{3}}{24 R^{2}}(\kappa-1)^{2} d s_{0},
\end{aligned}
$$


and $\overline{\mathcal{E}}$ is the energy of deformation per unit length of the initial configuration of the ring. Assuming that the reaction force of the substrate derives from a potential $\int_{0}^{2 \pi R} \bar{W} d s_{0}$, the equilibrium states of the ring are the solutions of

$$
\delta \mathcal{E}_{S}+\delta \mathcal{E}_{B}-\int_{0}^{2 \pi R} \delta \bar{W} d s_{0}=0
$$

where $\delta \mathcal{A}$ is the variation of quantity $\mathcal{A}$, for an arbitrary displacement field $R(\delta v, \delta u)$, which is $2 \pi$ periodic. The computation of the variations in Eq. (6) leads to the Euler-Lagrange equations for the equilibrium of the ring,

$$
\begin{aligned}
& \frac{\partial \overline{\mathcal{E}}}{\partial v}-\left(\frac{\partial \overline{\mathcal{E}}}{\partial v^{\prime}}\right)^{\prime}+\left(\frac{\partial \overline{\mathcal{E}}}{\partial v^{\prime \prime}}\right)^{\prime \prime}-\frac{\partial \delta \bar{W}}{\partial \delta v}=0 \\
& \frac{\partial \overline{\mathcal{E}}}{\partial u}-\left(\frac{\partial \overline{\mathcal{E}}}{\partial u^{\prime}}\right)^{\prime}+\left(\frac{\partial \overline{\mathcal{E}}}{\partial u^{\prime \prime}}\right)^{\prime \prime}-\frac{\partial \delta \bar{W}}{\partial \delta u}=0
\end{aligned}
$$

along with static boundary conditions that are naturally satisfied due to the $2 \pi$ periodicity condition on the displacements $v$ and $u$. All derivative terms in Eq. $7 \mathrm{~b}$ are explicitly reported in Appendix Appendix A.

\subsection{Asymptotic expansion and reactive force of the substrate}

We seek a solution of Eq. (7) as an expansion of the form

$$
\begin{aligned}
& v=v_{0}+\varepsilon A \sin (m \theta)+O\left(\varepsilon^{2}\right), \\
& u=\varepsilon B \cos (m \theta)+O\left(\varepsilon^{2}\right),
\end{aligned}
$$

where $\left(v_{0}, 0\right)$ corresponds to a radial pre-buckling deformation and $\varepsilon(A \sin (m \theta), B \cos (m \theta))$ represents an instability of azimuthal wavenumber $m$. From the requirement of $2 \pi$ periodic functions $v$ and $u, m$ has to be an integer. Also, we consider $m>1$ since $m=1$ corresponds to a solid body translation of the ring. The instability displacement field has amplitudes $\varepsilon A$ and $\varepsilon B$, where $\varepsilon$ is a small parameter.

Before substituting Eq. (8) into Eq. (7) and solving at each order in $\varepsilon$, we first need to determine the work, $\delta \bar{W}$, done by the reaction force, $\overline{\mathbf{F}}=-\left(\sigma \mathbf{e}_{\perp}+\tau \mathbf{e}_{\|}\right)$, that the substrate exerts on the ring. The normal stress $\sigma$ and the tangential stress $\tau$ at the interface between the ring and the substrate are computed by solving the corresponding two-dimensional elasticity problem using an Airy function, with pressure $P$ at $r=R_{0}$ and the displacement field given by Eq. (8), at $r=R$.

It is worth to note that in our computation, we enforce the continuity of the displacement and stress fields at the interface ring/substrate, similarly to Mei et al. (2011). This is a main difference with previous studies of wrinkling surfaces, e.g. in Seide (1962), who assumed zero shear stress and continuity of the normal stress. Such an approach yields a stretching energy in the ring much larger than the bending energy, in contradiction to what is expected in the wrinkling of a thin film (Audoly and Boudaoud, 2008a). Our explicit solution for the boundary value problem of the substrate is reported in Appendix B.

In short, we find that the stresses $\sigma$ and $\tau$ at the interface are

$$
\begin{aligned}
& \frac{\sigma}{\overline{\overline{E_{F}}}}=k_{0} v_{0}+k \varepsilon A \sin (m \theta)-\gamma p, \\
& \frac{\tau}{\overline{E_{F}}}=\mu \varepsilon B \sin (m \theta),
\end{aligned}
$$


with the following governing parameters

$$
\begin{aligned}
k_{0} & =\frac{\left(1-\nu_{S}\right)\left(1-\beta^{2}\right)}{1-2 \nu_{S}+\beta^{2}} \frac{1}{\xi}, \\
k & =\frac{\left(1-\nu_{S}\right)}{2}\left(S_{A}+S_{B} \frac{B}{A}\right) \frac{1}{\xi}, \\
\mu & =\frac{\left(1-\nu_{S}\right)}{2}\left(S_{B} \frac{A}{B}+T_{B}\right) \frac{1}{\xi}, \\
\gamma & =\frac{2 \beta^{2}\left(1-\nu_{S}\right)}{1-2 \nu_{S}+\beta^{2}} .
\end{aligned}
$$

Here, $k_{0}, k$, and $\mu$ are the dimensionless pre-wrinkling, wrinkling and shear stiffnesses of the substrate, respectively. The coefficient $\gamma$ quantifies the effect of pressure, $p$, on the ring through the term $-\gamma p$ in Eq. (9a). The value of $\gamma$ is smaller than one, reflecting the fact that the transmitted pressure decreases within the substrate. There are however two exceptions for which $\gamma=1$ : the limit where there is no substrate, $\beta \rightarrow 1$, and the limit of a perfectly incompressible substrate, $\nu_{S} \rightarrow 0.5$, in which the volumetric pressure remains constant throughout the substrate. The quantities $S_{A}, S_{B}$ and $T_{B}$ used in Eq. (10) are functions of both the cavity size $\beta$ and the azimuthal wavenumber $m$, and their full expressions are reported in Appendix B.

From the solution of the linear elasticity problem for the substrate (see Appendix B), the reaction force, $\mathbf{F}$, has a constant direction along $\mathbf{e}_{r}$. However, based on physical intuition, one would expect the restoring force to change its direction as the ring deforms. Accounting for this scenario in full would have required solving the elasticity problem for the substrate, with nonlinear kinematics. Here, for simplicity, we assume that the magnitude of the restoring force is given by the solution of the linear elasticity problem, while the direction of the force is given by the vectors $\mathbf{e}_{\perp}$ and $\mathbf{e}_{\|}$. Depending on which configuration of the ring is used (initial or deformed), two cases need to be considered to define the normal and tangential vectors $\mathbf{e}_{\perp}$ and $\mathbf{e}_{\|}$, respectively. If we define these vectors from the initial configuration, then $\left(\mathbf{e}_{\perp}, \mathbf{e}_{\|}\right)=\left(\mathbf{e}_{\mathbf{r}}, \mathbf{e}_{\theta}\right)$, so that the reaction force $\mathbf{F}$ keeps a constant direction while the ring deforms. If we use the deformed configuration, then $\mathbf{F}$ is modeled as a follower force whose direction changes with the ring deformation. In this case, $\left(\mathbf{e}_{\perp}, \mathbf{e}_{\|}\right)=(\mathbf{N}, \mathbf{T})$, where $\mathbf{N}$ is the inward normal vector orthogonal to the ring center-line, in its deformed state. In order to take both of these options into account in the same model, we define a parameter, $\chi$, which can take the values $\chi=1$ or $\chi=0$, when either the undeformed or deformed configurations are used. Thus, we express the reaction force and its elementary work per unit length of the ring as

$$
\begin{aligned}
\mathbf{F} & =-\left[\left(\sigma \mathbf{e}_{\mathbf{r}}+\tau \mathbf{e}_{\theta}\right) \chi+(\sigma \mathbf{N}+\tau \mathbf{T})(\chi-1)\right], \\
\delta \bar{W} & =\mathbf{F} \cdot R\left(\delta v \mathbf{e}_{\mathbf{r}}+\delta u \mathbf{e}_{\theta}\right) .
\end{aligned}
$$

where $\cdot$ is the Euclidean dot product.

\subsubsection{The principal solution: order 0 in $\varepsilon$}

We now proceed to obtain the principal solution and the critical instability modes by substituing Eq. (8) into Eq. (7) and solving the resulting equations for each order of $\varepsilon$. At order 0, Eq. (3) for the stretching deformation $\eta$ yields $\eta_{0}=v_{0}=\sigma_{0} / \overline{E_{F}}$ and the linear approximation of the Euler-Lagrange Eq. (7a)

$$
v_{0}=\frac{\gamma p}{h+h^{3} / 12+k_{0}}=\frac{\sigma_{0}}{\overline{E_{F}}}
$$

relates the dimensionless pressure, $p$, and the dimensionless hoop stress, $\sigma_{0} / \overline{E_{F}}$, in the ring. Note that Eq. (7b) is automatically satisfied at order 0 in $\varepsilon$. In the absence of a substrate, the ratio $\sigma_{0} / P$ reads

$$
\lim _{\beta \rightarrow 1}\left(\frac{\sigma_{0}}{P}\right)=\frac{1}{h}+O(h)
$$


and in the limit of the ring that is much stiffer than the substrate we obtain

$$
\lim _{\xi \rightarrow \infty}\left(\frac{\sigma_{0}}{P}\right)=\frac{\gamma}{h}+O(h),
$$

so that $\frac{\sigma_{0}}{P}<\lim _{\xi \rightarrow \infty}\left(\frac{\sigma_{0}}{P}\right) \leq \lim _{\beta \rightarrow 1}\left(\frac{\sigma_{0}}{P}\right)$. For $\nu_{S}=0.5$, we have $\gamma=1$ through the definition in Eq. $10 \mathrm{~d}$ ) and both limits in Eqs. (13) and (14) are equal. Thus, the hoop stress in a very stiff ring lying on a soft incompressible substrate is the same as the hoop stress in a ring with no substrate, which serves as a verification of the rationale thus far.

\subsubsection{The instability: order 1 in $\varepsilon$}

At order 1 in $\varepsilon$, the Euler-Lagrange Eqs. (7) write

$$
\begin{aligned}
& \left(a_{1} p+\widetilde{a_{1}}\right) A+\left(b_{1} p+\widetilde{b_{1}}\right) B=0, \\
& \left(a_{2} p+\widetilde{a_{2}}\right) A+\left(b_{2} p+\widetilde{b_{2}}\right) B=0,
\end{aligned}
$$

where $a_{i}, \widetilde{a_{i}}, b_{i}$ and $\widetilde{b_{i}}$ are functions of the azimuthal wavenumber $m$, the stiffness ratio $\xi$, the cavity size $\beta$ and the substrate Poisson's ratio $\nu_{S}$, reported in full in Appendix C. The linear system in Eq. 15) has a nontrivial solution when its determinant vanishes, which for a given value of $m$ occurs at the dimensionless pressure

$$
p_{m}=\frac{P_{m}}{\overline{E_{F}}}=\frac{-\widetilde{a_{1}} \widetilde{b_{2}}+\widetilde{b_{1}} \widetilde{a_{2}}}{a_{1} \widetilde{b_{2}}+\widetilde{a_{1}} b_{2}-b_{1} \widetilde{a_{2}}-\widetilde{b_{1}} a_{2}} .
$$

The critical azimuthal wavenumber and the dimensionless critical pressure can now be obtained from Eq. 16 . by minimizing over all possible values of $m$

$$
\left[m_{c}, p_{c}\right]=\min _{m=2,3, \ldots}\left(p_{m}\right)
$$

For the general case, this minimization must be performed numerically. However, in the absence of the substrate, we obtain that $m_{c}=2$ and Eq. (16) simplifies to

$$
\lim _{\beta \rightarrow 1}\left(p_{c}\right)=\lim _{\beta \rightarrow 1}\left(p_{2}\right)=-\frac{1}{4-\chi} h^{3}-\frac{4}{(4-\chi)^{2}} h^{5}+O\left(h^{7}\right) .
$$

The term of order $h^{3}$ corresponds to the classical dimensionless critical pressure for a ring with no substrate (Lévy, 1884, Carrier, 1947, Timoshenko and Gere, 1961, Combescure, 1981). The term in $h^{5}$ is a correction also reported by Atanackovic (1998). In the limit of a ring much stiffer than the substrate, we also have $m_{c}=2$, with

$$
\lim _{\xi \rightarrow \infty}\left(p_{c}\right)=\lim _{\xi \rightarrow \infty}\left(p_{2}\right)=-\frac{1}{2} \frac{\left(-1+2 \nu_{S}-\beta^{2}\right) h^{3}}{\beta^{2}\left(-1+\nu_{S}\right)(4-\chi)}-\frac{2\left(-1+2 \nu_{S}-\beta^{2}\right) h^{5}}{\beta^{2}\left(-1+\nu_{S}\right)(4-\chi)^{2}}+O\left(h^{7}\right),
$$

so that $\lim _{\xi \rightarrow \infty}\left(p_{c}\right) \leq \lim _{\beta \rightarrow 1}\left(p_{c}\right)<0$. Again, for $\nu_{S}=0.5$, we observe that the limits equal, corroborating the physical intuition that as the stiffness ratio $\xi$ increases, the effect of the substrate becomes negligible.

\subsection{Critical stress, wavelength and comparison to wrinkling on a planar substrate}

To determine the effect of curvature on the instability, we compare the critical hoop stress, $\sigma_{c}$, in the ring, given by Eq. 12 with $p=p_{c}$, as well as the wavelength $\lambda_{c}=2 \pi R / m_{c}$ of the wrinkling mode, against their 
counterparts for an initially planar film on a plane substrate of infinite thickness (Chen and Hutchinson, 2004)

$$
\begin{aligned}
& \sigma_{\text {Plane }}=\frac{\overline{E_{F}}}{4}\left(3 \frac{{\overline{E_{S}}}_{\overline{E_{F}}}^{*}}{{ }^{2 / 3}}=\frac{\overline{E_{F}}}{4}\left(\frac{4\left(1-\nu_{S}\right)^{2}}{3-4 \nu_{S}} \frac{3}{\xi}\right)^{2 / 3},\right. \\
& \lambda_{\text {Plane }}=2 \pi H\left(\frac{1}{3} \frac{\overline{E_{F}}}{{\overline{E_{S}}}^{*}}\right)^{1 / 3}=2 \pi H\left(\frac{1}{3} \frac{3-4 \nu_{S}}{4\left(1-\nu_{S}\right)^{2}} \xi\right)^{1 / 3},
\end{aligned}
$$

where ${\overline{E_{S}}}^{*}=4 \overline{E_{S}}\left(1-\nu_{S}\right)^{2} /\left(3-4 \nu_{S}\right)$ is the effective stiffness of the substrate. Together, Eqs. 20 and 12 yield

$$
\begin{aligned}
\frac{\sigma_{c}}{\sigma_{\text {Plane }}} & =\frac{4 \gamma p_{c}}{h+h^{3} / 12+k_{0}}\left(\frac{12\left(1-\nu_{S}\right)^{2}}{3-4 \nu_{S}} \frac{1}{\xi}\right)^{-2 / 3}, \\
\frac{\lambda_{c}}{\lambda_{\text {Plane }}} & =\frac{1}{h m_{c}}\left(\frac{3-4 \nu_{S}}{12\left(1-\nu_{S}\right)^{2}} \xi\right)^{-1 / 3},
\end{aligned}
$$

and, in the limit of absence of the substrate, Eq. 21a reduces to

$$
\lim _{\beta \rightarrow 1}\left(\frac{\sigma_{c}}{\sigma_{\text {Plane }}}\right)=\frac{1}{\sigma_{\text {Plane }}} \lim _{\beta \rightarrow 1}\left(\sigma_{c}\right)=\frac{1}{\sigma_{\text {Plane }}} \lim _{\beta \rightarrow 1}\left(\sigma_{2}\right)=\frac{4}{4-\chi}\left(\frac{12\left(1-\nu_{S}\right)^{2}}{\left(3-4 \nu_{S}\right) \xi}\right)^{-2 / 3} h^{2}+O\left(h^{4}\right),
$$

where $\nu_{S}$ is the Poisson's ratio of the substrate in the planar case.

In $\S 5.4$, we shall make use of Eqs. 211) and Eq. 222) to further quantify the sensitivity of the stress and wavelength to the curvature of the substrate.

\section{Numerical simulations}

In $\S 5$, we will contrast the predictions from the above analysis with the results of a series of finite element simulations performed using the commercial package Abaqus, with the BUCKLE analysis, which provides the buckling load and the corresponding eigenmodes.

As in the analytical study, the cylindrical structure was modeled as an annulus of a soft substrate with a stiffer thin film adhered to its exterior, under plane strain conditions. The bonding between the substrate and the film is assumed to be perfect, such that both share nodes. Negative pressure is applied on the interior surface, to model the effect of the pressure differential between the inner surface of the annulus (the cavity) and the exterior of the system. The buckling analysis provides the value of the critical pressure, $p_{c}$, as well as the corresponding critical mode. Rigid body motions are removed by constraining the displacement of two points on the film.

Both substrate and film are modeled as incompressible linearly elastic materials, $\nu_{S}=\nu_{F}=0.5$. These results were compared to additional simulations using a Neo-Hookean model but no difference was observed given the low values of strain involved. The substrate was modeled using quadrilateral plane strain elements. Due to the incompressibility, the corresponding hybrid element, CPE4H, was used. The film was modeled using B21 beam elements. In order to account for the effect of plane strain, the stiffness of the beam is defined as $E_{F} /\left(1-\nu_{F}^{2}\right)$.

All of the results presented were obtained using 1000 elements in the circumferential direction, and $150\left(R-R_{0}\right)$ elements in the radial direction. The mesh size was validated with a convergence analysis. By way of example, differences less than $0.5 \%$ in critical pressure were obtained when comparing results for a mesh with twice the elements in each direction, even in the cases of wrinkling with the shorter wavelengths. The deviations between the two meshes were, however, larger $(\sim 5 \%)$ for the case when the critical buckling 
mode is a Biot instability, due to the infinite number of wavelengths associated to the same buckling mode. However, as stated in $\S 2$ this mode shall not be studied in detail as we focus on the wrinkling and global buckling loads.

To test the validity of using beam theory to describe the ring in our problem, we also performed numerical simulations with 2D solid elements (CPE4H, the same used for the substrate) and found excellent agreement with the simulations using beam elements. However, in order to achieve such agreement, the mesh needs to be greatly refined, resulting in a significant increase in computational cost. Attempts to use a mesh size similar to that of our previous simulations showed clear disagreement. Given the excellent agreement between the two versions - either using CPE4H (with a fine mesh) or B21 for the ring - as well as the significantly lower computational cost of the B21 elements, we have decided to use beam elements for the ring in our analysis.

\section{Results}

Having introduced our analytical and numerical methods, we now present the results of a systematic exploration of the mechanical response of our system for different geometric and material parameters. Throughout, we provide a direct comparison between analytical results and numerical simulations, finding good agreement. A few instances of discrepancy will also be discussed.

For the geometric parameters, we have varied the cavity size, $\beta=R_{0} / R$, and the dimensionless thickness, $h=H / R$. Three representative values were chosen for $\beta$ (depicted in the insets of Fig. 3 a-c): a small cavity, $\beta=0.2$; a cavity with size half of the external radius, $\beta=0.5$; and a large cavity, $\beta=0.8$. Moreover, $h$ was varied in the range $10^{-3}$ to $10^{-1}$. This parameter has two different physical interpretations. On one hand, for a substrate with given curvature, i.e. fixed $R$, increasing $h$ is equivalent to increasing the thickness of the ring. On the other hand, for a ring of given thickness $H$, the value of $h$ decreases with the curvature, $1 / R$.

For the material properties, we have considered values for the stiffness ratio between the film and the substrate, $\xi=\bar{E}_{F} / \bar{E}_{S}$, spanning over five orders of magnitude, from $10^{2}$ to $10^{7}$. The substrate is taken to be incompressible, $\nu_{S}=0.5$, since most of the relevant experiments that have motivated our study (Bowden et al., 1998, Huck et al., 2000, Yu and Jiang, 2010, Terwagne et al., 2014) use nearly incompressible elastomeric substrates. Moreover, it is important to note that, even if the analytical model has been derived assuming a general value of $\nu_{S}$, it is expected to be less accurate for increasing deviations from incompressibility. This was analyzed by Cai et al. (2011), who showed that for the wrinkling of plates deviations when $\nu_{S}=0.3$ are of just a few percent.

In the presentation of our results, we first consider the effect of $h$ and $\xi$ on the hoop-stress and on the critical pressure. Then, we rationalize the transition from wrinkling to global buckling, shown in Fig. 1. We finally compare the critical stress and wavelength of the wrinkling mode to their planar substrate counterparts and discuss the effect of curvature.

\subsection{Hoop stress prior to wrinkling}

In Fig. 3, we plot the hoop stress in the ring, normalized by the pressure, $\sigma_{0} / P$, as a function of the dimensionless thickness, $h=H / R$. We find that the hoop stress decreases monotonically with the dimensionless thickness, and increases with both the stiffness ratio and the cavity size. When the ring is much stiffer than the substrate (i.e. $\xi \rightarrow \infty$ ), the hoop stress scales as $\sigma_{0} / P \sim(H / R)^{-1}$, with a prefactor given by Eq. (14). For a given thickness of the ring and a given cavity size, the hoop stress in the ring decreases as the curvature of the substrate increases. This observation is consistent with the classic result for the hoop stress, $\sigma_{0}=P R / H$, for a depressurized thin-walled cylindrical pressure vessel. This result can be recovered from Eq. (12) by taking $\gamma=1, k_{0}=0$ and performing a Taylor expansion in $h$, about 0 .

\subsection{Critical pressure}

In Fig. 4, we plot the dimensionless critical pressure, $\left|p_{c}\right|=\left|P_{c}\right| / \overline{E_{F}}$, as a function of the dimensionless thickness, $h$, and observe two different regimes. For low values of $h$, the ring wrinkles with an azimuthal 
wavenumber $m_{c} \gg 2$, with a critical pressure $\left|p_{c}\right|$ that increases with $h$. When $h$ reaches a threshold value, $h^{*}$, the wavenumber decreases suddenly to $m_{c}=2$; the nature of the instability changes from a wrinkling to a global buckling mode. In this regime, there is a clear asymptote as $\xi \rightarrow \infty$, given by Eq. (19), which corresponds to the buckling of a ring with no substrate.

Depending on the model used for the reaction force of the substrate (constant direction force, $\chi=1$, or follower force, $\chi=0$, see $\S 3.2$, Eq. (11) yields two different analytical predictions, shown in Fig. 4 as a solid line for $\chi=0$, and a dashed line for $\chi=1$. We note that the agreement between FEM simulations and analytical predictions is superior for $\chi=0$, in particular for the global mode. In other words, it is better to model the reaction force of the substrate as a pressure field which remains normal to the ring center-line $C$ as it deforms, than as a force with constant direction. Thus, from now on, all analytical results will be only presented for $\chi=0$.

Despite the overall good agreement between the analytical and numerical results, there are noticeable discrepancies in the global modes for low values of the stiffness ratio. The reason is that, for $\xi=10^{2}$, the strains in the substrate can become significant, such that the assumptions for our linear theory are no longer valid (see Appendix B). A particularly extreme case is the appearance of Biot modes for $\beta=0.2$ and $\xi=10^{2}$. These modes, although possible, only occur in a small region of our parameter space and, as mentioned above, are beyond the scope of this work.

In short, our model exhibits limitations if the ring and the substrate have comparable stiffness, or when there is a Biot mode (small cavity). Apart from this extreme combination of parameters, rarely observed in experimental configurations, the model performs successfully.

\subsection{Phase diagram}

We proceed by focusing on the transition from wrinkling to global buckling, towards first constructing a phase diagram in the $(\xi, h)$ parameter space and then quantifying the dependence of the boundary, $h^{*}(\xi)$, between the two modes on the size of the cavity, $\beta$. Given that we do not have a closed form expression for $h^{*}$, we use a numerical method which tracks any jump from $m_{c}=2$ to $m_{c}^{*}>2$ in Eq. (17), when $h$ is decreased.

In Fig. 5(a), we plot $h^{*}$ as a function of $\xi$, for $\beta=0.5$. The boundary between modes is consistent with a power-law, $h^{*} \sim \xi^{-3}$, which divides the phase diagram into a wrinkling domain $\left(h<h^{*}\right)$ and a global buckling domain $\left(h>h^{*}\right.$, shaded region). Again, there is good agreement between numerical and analytical solutions.

In Fig. 5(b), we extend the phase diagram to cavity sizes from $\beta=0.2$ to $\beta=0.8$. We predict that the power-law with exponent -3 , mentioned above for $\beta=0.5$, is still valid as $\beta$ increases, even if there are some deviations towards the higher values. To quantify the appropriateness of the -3 power-law, we fit a curve of the form $\xi=f_{2} h^{f_{1}}$ to the analytically calculated boundaries. In Fig. 5(c) we plot the fitting parameters $f_{1}$ and $f_{2}$ as a function of $\beta$. We find that the exponent is $f_{1} \approx-3$ for $\beta<0.7$, and then decreases rapidly to a value of $f_{1} \approx-4.25$ for $\beta=0.8$. The decrease of the prefactor $f_{2}$ is more pronounced and reflects the fact that $h^{*}$ decreases as $\beta$ increases.

The evolution of $m_{c}^{*}$ (i.e. azimuthal wavenumber of the wrinkling mode for $h=h^{*}$ ) as a function of $\beta$ and $\xi$ is presented in Fig. 5(d). We observe that $m_{c}^{*}$ increases with $\beta$, while it is nearly insensitive to $\xi$. For example, for $\beta=0.5$, we find that $m_{c}^{*}=9$ for $\xi>10^{2}$ and $m_{c}^{*}=10$ for $\xi=10^{2}$. Predictions for $\xi>10^{2}$ are in relatively good agreement with finite element simulations, which have shown that $m_{c}^{*}=10$. For $\xi=10^{2}$, FEM simulations yield $m_{c}^{*}=15$, pointing out, once again, the limitation of our analytical approach in the limit when the stiffness of the ring and the substrate become comparable.

\subsection{Comparison of wrinkling in our curved system with that on infinite planar substrate}

Thus far, we have shown that the ring may wrinkle or buckle globally, depending on the curvature and stiffness of the substrate. We now focus on the wrinkling mode of the ring, with the aim of comparing the critical stress and wavelength of our curved system to their counterparts for a infinite planar substrate. We shall center our discussion of this comparison for $\beta=0.5$. In Appendix $\mathrm{D}$, we report the results for $\beta=0.2$ and $\beta=0.8$, which are qualitatively similar. 


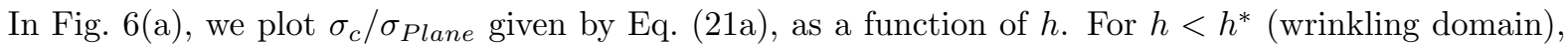
we find that $\sigma_{c} / \sigma_{\text {Plane }} \approx 1$, in agreement with the FEM simulations. To further quantify how close to unity is this ratio, in Fig. 6(b) we plot the dimensionless critical normal force $\sigma_{c} h / \overline{E_{F}}$ as a function of the planar result, $\sigma_{\text {Plane }} h / E_{F}$. We obtain a line with unit slope, indicating that the substrate curvature has no significative effect on the critical stress for wrinkling. From this observation, and using Eq. (12) with $\sigma_{0}=\sigma_{\text {Plane }}$, we write the following approximation for the dimensionless critical pressure,

$$
\left|p_{c}\right|=\frac{h+k_{0}}{\gamma} \frac{\sigma_{\text {Plane }}}{\overline{E_{F}}}+O\left(h^{3}\right)
$$

with $k_{0}$ and $\gamma$ given by Eq. (10). Finally, back to Fig. 6(a), for $h>h^{*}$ (global buckling domain), we find that $\sigma_{c} / \sigma_{\text {Plane }}$ first decreases with $h$, then reaches a local minimum and eventually increases as a power-law with slope 2, towards the asymptotic limit given by Eq. 22 . The evolution of $\sigma_{c} / \sigma_{\text {Plane }}$ in the global buckling domain is also reproduced well by FEM simulations.

We now investigate the effect of curvature on the wavelength of the instability mode. In Fig. 7(a), we plot $\lambda_{c} / \lambda_{\text {Plane }}$, given by Eq. $(21 \mathrm{~b})$, as a function of $h$. Focusing on the wrinkling domain, we find that $\lambda_{c} / \lambda_{\text {Plane }} \approx 1$, in agreement with FEM simulations. In Fig. $7(\mathrm{~b})$, once again, we quantify how close to unity this ratio is by plotting the critical wavelength $\lambda_{c}$ as a function of the planar result, $\lambda_{\text {Plane }}$. To first approximation, noting the large dynamic range (at least two orders of magnitude) in both axes of the plot, we find a line with unit slope that passes through the origin, suggesting that the substrate curvature has no significative effect on the wavelength of the wrinkling mode. The only deviations arise from the discrete nature of the wavenumber, since the geometry of the ring enforces $2 \pi$ periodic wrinkling modes. To highlight this phenomenon, in Fig. 7(c), we plot the critical azimuthal wavenumber $m_{c}$, given by Eq. (17), as a function of $h$ and we superimpose the planar substrate result (dashed line), $m_{\text {Plane }}=2 \pi R / \lambda_{\text {Plane }}$. We find that $m_{c}$ is a decreasing stair function of $h$. The deviation in wavelength between the curved and planar cases scales as $\left(\left|\lambda_{c}-\lambda_{\text {Plane }}\right|\right) / \lambda_{\text {Plane }} \sim 1 / m$, which is maximum for $m_{c}^{*}$. As shown previously in Fig. $5(\mathrm{~d}), m_{c}^{*}$ increases with $\beta$, hence the deviation $\left(\left|\lambda_{c}-\lambda_{\text {Plane }}\right|\right) / \lambda_{\text {Plane }}$ is maximum for a small cavity size. By way of example, for $\beta=0.5$, Fig. 5(d) indicates that $m_{c}^{*}=9$, leading to a maximum deviation $\left(\left|\lambda_{c}-\lambda_{\text {Plane }}\right|\right) / \lambda_{\text {Plane }} \approx 10 \%$, that rapidly decreases as $m$ increases.

\section{Conclusion}

We have considered the two-dimensional problem of a ring bound to an elastic substrate which contains a cavity that is depressurized. An energy formulation was used to derive the Euler-Lagrange equations that govern the equilibrium of the ring, and solved them via an asymptotic expansion. As an improvement to previous results in the literature, our analytical approach accounts for the effect of curvature in modeling the reaction force of the substrate. These analytical results were compared with numerical simulations.

We first studied the principal solution, obtaining an expression for the hoop stress in the ring as a function of the applied pressure. We then performed a stability analysis of the problem to determine the critical pressure, $P_{c}$, and the corresponding instability mode. Depending on the dimensionless thickness and stiffness ratio ( $h$ and $\xi$ ) we have identified two different regimes: local wrinkling of the ring, and global buckling of the structure. The boundary between both regions of instability was described via a detailed phase diagram, which quantifies the value of $h$ and $\xi$ at which the transition between instabilities occurs and takes into account the cavity size, $\beta$. Our results can be used as a design guideline to target a desired mode. Finally, we have shown that the critical stress for wrinkling and the resulting wavelength do not depend significantly on the curvature of the substrate. However, curvature imposes a discretization of the wrinkling wavelength due to the periodic closing conditions of the ring.

Our study focused on a 2D curved system which exhibits instability modes analogous to the cylindrical pattern found for uniaxial compression of a film on a flat infinite substrate. Considering more complex loading conditions (e.g. also introducing axial loading) or shells with non-zero Gaussian curvature, should lead to more complex patterns that deserve to be investigated further. Having validated the FEM analysis, as well as carefully considering the elastic response of the curved substrate, extending the study to these other scenarios and addressing the issue of pattern selection is an exciting avenue for future research. 


\section{Acknowledgements}

D.T. thanks the Belgian American Education Foundation (B.A.E.F.), the Fulbright Program and the Wallonie-Bruxelles International Excellence Grant WBI World. M.B. thanks the Fulbright Program. P.M.R. is grateful to financial support from the National Science Foundation, CMMI-1351449 (CAREER) and SaintGobain. 


\section{Appendix A. Derivative terms in Euler-Lagrange equation}

The terms in the Euler-Lagrange equation for the equilibrium of the film, Eq. 7b, are

$$
\begin{aligned}
& \frac{\partial \overline{\mathcal{E}}}{\partial v}=-\frac{h^{2}}{12}\left(-2 v^{2}+\left(2-4 v^{\prime \prime}\right) v+2 v^{\prime \prime}-{v^{\prime}}^{2}+2 u u^{\prime \prime}-4 u^{\prime} v^{\prime \prime}-2 v^{\prime} u^{\prime \prime}+2{u^{\prime}}^{2}+u^{2}\right)\left(v^{\prime \prime}+v-\frac{1}{2}\right) \\
& +\frac{1}{2}\left(v^{2}+\left(2+2 u^{\prime}\right) v+u^{\prime 2}+2 u^{\prime}+\left(u-v^{\prime}\right)^{2}\right)\left(1+v+u^{\prime}\right) \\
& \frac{\partial \overline{\mathcal{E}}}{\partial u}=\frac{h^{2}}{24}\left(-2 v^{2}+\left(2-4 v^{\prime \prime}\right) v+2 v^{\prime \prime}-v^{\prime 2}+2 u u^{\prime \prime}-4 u^{\prime} v^{\prime \prime}-2 v^{\prime} u^{\prime \prime}+2 u^{\prime 2}+u^{2}\right)\left(u+u^{\prime \prime}\right) \\
& +\frac{1}{2}\left(u^{2}-2 u v^{\prime}+v^{\prime 2}+\left(v+2+u^{\prime}\right)\left(v+u^{\prime}\right)\right)\left(u-v^{\prime}\right) \\
& \left(\frac{\partial \overline{\mathcal{E}}}{\partial v^{\prime}}\right)^{\prime}=-\frac{h^{2}}{12}\left(\left(-2 v+1-3 v^{\prime \prime}-u^{\prime \prime \prime}\right) v^{\prime}+\left(3 u^{\prime \prime}-2 v^{\prime \prime \prime}+u\right) u^{\prime}-3 u^{\prime \prime} v^{\prime \prime}+(1-2 v) v^{\prime \prime \prime}+u u^{\prime \prime \prime}\right)\left(v^{\prime}+u^{\prime \prime}\right) \\
& -\frac{h^{2}}{24}\left(\left(-4 v+2-4 u^{\prime}\right) v^{\prime \prime}-2 v^{2}+2 v-2 v^{\prime} u^{\prime \prime}-v^{\prime 2}+2 u u^{\prime \prime}+2{u^{\prime}}^{2}+u^{2}\right)\left(v^{\prime \prime}+u^{\prime \prime \prime}\right) \\
& -\left(u-v^{\prime}\right)\left(\left(v+1+v^{\prime \prime}\right) v^{\prime}+\left(u+u^{\prime \prime}\right) u^{\prime}+(1+v) u^{\prime \prime}-u v^{\prime \prime}\right) \\
& -\frac{1}{2}\left(u^{\prime}-v^{\prime \prime}\right)\left(u^{\prime 2}+(2 v+2) u^{\prime}+v^{2}+2 v+\left(u-v^{\prime}\right)^{2}\right) \\
& \left(\frac{\partial \overline{\mathcal{E}}}{\partial u^{\prime}}\right)^{\prime}=\frac{h^{2}}{6}\left(u^{\prime}-v^{\prime \prime}\right)\left(\left(-2 v+1-3 v^{\prime \prime}-u^{\prime \prime \prime}\right) v^{\prime}+\left(3 u^{\prime \prime}-2 v^{\prime \prime \prime}+u\right) u^{\prime}-3 u^{\prime \prime} v^{\prime \prime}+(1-2 v) v^{\prime \prime \prime}+u u^{\prime \prime \prime}\right) \\
& +\frac{h^{2}}{12}\left(\left(2 u-2 v^{\prime}\right) u^{\prime \prime}+\left(-4 v+2-4 u^{\prime}\right) v^{\prime \prime}-2 v^{2}+2 v+2{u^{\prime}}^{2}-v^{\prime 2}+u^{2}\right)\left(-v^{\prime \prime \prime}+u^{\prime \prime}\right) \\
& +\left(\left(u+u^{\prime \prime}\right) u^{\prime}+\left(v^{\prime}+u^{\prime \prime}\right) v+u^{\prime \prime}+\left(1+v^{\prime \prime}\right) v^{\prime}-u v^{\prime \prime}\right)\left(1+v+u^{\prime}\right) \\
& +\frac{1}{2}\left(v^{\prime}+u^{\prime \prime}\right)\left(u^{\prime 2}+(2 v+2) u^{\prime}+v^{2}+2 v+\left(u-v^{\prime}\right)^{2}\right) \\
& \left(\frac{\partial \overline{\mathcal{E}}}{\partial v^{\prime \prime}}\right)^{\prime \prime}=-\frac{h^{2}}{6}\left(v-\frac{1}{2}+u^{\prime}\right)\left(-3 v^{\prime \prime 2}+\left(1-2 v-4 u^{\prime \prime \prime}\right) v^{\prime \prime}+u^{\prime 2}+\left(-2 v^{\prime \prime \prime \prime}+4 u^{\prime \prime \prime}\right) u^{\prime}+(1-2 v) v^{\prime \prime \prime \prime}\right) \\
& -\frac{h^{2}}{6}\left(v-\frac{1}{2}+u^{\prime}\right)\left(3 u^{\prime \prime 2}+\left(-5 v^{\prime \prime \prime}+u\right) u^{\prime \prime}-2{v^{\prime}}^{2}+\left(-5 v^{\prime \prime \prime}-u^{\prime \prime \prime \prime}\right) v^{\prime}+u u^{\prime \prime \prime \prime}\right) \\
& -\frac{h^{2}}{3}\left(\left(-2 v+1-3 v^{\prime \prime}-u^{\prime \prime \prime}\right) v^{\prime}+\left(-2 u^{\prime}+1-2 v\right) v^{\prime \prime \prime}+\left(3 u^{\prime \prime}+u\right) u^{\prime}+u u^{\prime \prime \prime}-3 u^{\prime \prime} v^{\prime \prime}\right)\left(v^{\prime}+u^{\prime \prime}\right) \\
& -\frac{h^{2}}{12}\left(\left(-4 v+2-4 u^{\prime}\right) v^{\prime \prime}-2 v^{2}+2 v-2 v^{\prime} u^{\prime \prime}-v^{\prime 2}+2 u u^{\prime \prime}+2{u^{\prime 2}}^{2}+u^{2}\right)\left(v^{\prime \prime}+u^{\prime \prime \prime}\right) \\
& \left(\frac{\partial \overline{\mathcal{E}}}{\partial u^{\prime \prime}}\right)^{\prime \prime}=\frac{h^{2}}{12}\left(u-v^{\prime}\right)\left(-3 v^{\prime \prime 2}+\left(1-2 v-4 u^{\prime \prime \prime \prime}\right) v^{\prime \prime}-2 v^{\prime 2}+\left(-5 v^{\prime \prime \prime}-u^{\prime \prime \prime \prime}\right) v^{\prime}+\left(-2 u^{\prime}+1-2 v\right) v^{\prime \prime \prime \prime \prime}\right) \\
& +\frac{h^{2}}{12}\left(u-v^{\prime}\right)\left(\left(u^{\prime \prime \prime \prime}+u^{\prime \prime}\right) u-5 u^{\prime \prime} v^{\prime \prime \prime}+3 u^{\prime \prime 2}+4 u^{\prime} u^{\prime \prime \prime}+u^{\prime 2}\right) \\
& +\frac{h^{2}}{6}\left(u^{\prime}-v^{\prime \prime}\right)\left(\left(-2 v+1-3 v^{\prime \prime}-u^{\prime \prime \prime}\right) v^{\prime}+\left(3 u^{\prime \prime}-2 v^{\prime \prime \prime}+u\right) u^{\prime}-3 u^{\prime \prime} v^{\prime \prime}+(1-2 v) v^{\prime \prime \prime}+u u^{\prime \prime \prime}\right) \\
& +\frac{h^{2}}{24}\left(\left(2 u-2 v^{\prime}\right) u^{\prime \prime}+\left(-4 v+2-4 u^{\prime}\right) v^{\prime \prime}-2 v^{2}+2 v+2{u^{\prime}}^{2}-v^{\prime 2}+u^{2}\right)\left(-v^{\prime \prime \prime}+u^{\prime \prime}\right)
\end{aligned}
$$


and

$$
\begin{aligned}
& \frac{\partial \delta \bar{W}}{\partial \delta v}=\frac{1}{2} \frac{2 \tau(-1+\chi)\left(-1+u^{\prime}+v\right)\left(u-v^{\prime}\right)-\sigma\left((-1+\chi) v^{\prime 2}-2 u(-1+\chi) v^{\prime}+2+(-1+\chi) u^{2}\right)}{h} \\
& \frac{\partial \delta \bar{W}}{\partial \delta u}=\frac{1}{2} \frac{-2 \sigma\left(-1+u^{\prime}+v\right)\left(u-v^{\prime}\right)(-1+\chi)-\tau\left((-1+\chi) v^{\prime 2}-2 u(-1+\chi) v^{\prime}+2+(-1+\chi) u^{2}\right)}{h}
\end{aligned}
$$

\section{Appendix B. Response of the substrate}

In this appendix we consider the boundary value problem of the substrate, subjected to the ring displacement $R(v(\theta), u(\theta)), v$ and $u$ given by Eq. (8), at the interface $r=R$ between the ring and the substrate and to the pressure $P$ at $r=R_{0}=\beta R$. The substrate is assumed to be in a state of plane strain. We note $\alpha=3-4 \nu_{S}$ and introduce the shear modulus $G=\overline{E_{S}}\left(1-\nu_{S}\right) / 2$. The components of the stress in the substrate are represented by $\sigma_{r r}, \sigma_{r \theta}$, and the displacement field is $\left(U_{r}, U_{\theta}\right)$. The $2 \mathrm{D}$ problem of elasticity is solved by finding an Airy function of the form (Michell, 1899),

$$
\phi_{m}(r, \theta)=B_{1} r^{2}+B_{2} \ln (r)+\varepsilon\left(A_{1} r^{m+2}+A_{2} r^{-m+2}+A_{3} r^{m}+A_{4} r^{-m}\right) \sin (m \theta),
$$

where $A_{i}$ and $B_{i}$ are unknown constants determined by the boundary conditions

$$
\begin{aligned}
\sigma_{r r}\left(R_{0}, \theta\right) & =-P, \\
\sigma_{r \theta}\left(R_{0}, \theta\right) & =0, \\
U_{r}(R, \theta) & =R v(\theta), \\
U_{\theta}(R, \theta) & =R u(\theta),
\end{aligned}
$$

which are assumed to apply at $r=R_{0}$ and $r=R$. The first two equations stand for the continuity of the stress at the boundary of the cavity, whereas the last two stand for the continuity of the displacement at the interface between the substrate and the ring.

The stress and displacement fields resulting from the Airy function Eq. (B.1) are $(\overline{B a r b e r}, 2002)$

$$
\begin{gathered}
\sigma_{r r}(r, \theta)=2 B_{1}+\frac{B_{2}}{r^{2}}+\left(\begin{array}{l}
-A_{1}(m+1)(m-2) r^{m}-A_{2}(m+2)(m-1) r^{-m} \\
-A_{3} m(m-1) r^{m-2}-A_{4} m(m+1) r^{-m-2}
\end{array}\right) \sin (m \theta), \\
\sigma_{r \theta}(r, \theta)=\left(\begin{array}{l}
-A_{1} m(m+1) r^{m}+A_{2} m(m-1) r^{-m} \\
-A_{3} m(m-1) r^{m-2}+A_{4} m(m+1) r^{-m-2}
\end{array}\right) \cos (m \theta), \\
U_{r}(r, \theta)=\frac{1}{2 G}\left[\begin{array}{l}
\left.B_{1}(\alpha-1) r-\frac{B_{2}}{r}+\left(\begin{array}{c}
A_{1}(\alpha-m-1) r^{m+1}+A_{2}(\alpha+m-1) r^{-m+1} \\
-A_{3} m r^{m-1}+A_{4} m r^{-m-1}
\end{array}\right) \sin (m \theta)\right]
\end{array}\right), \\
U_{\theta}(r, \theta)=\frac{1}{2 G}\left(\begin{array}{l}
-A_{1}(\alpha+m+1) r^{m+1}+A_{2}(\alpha-m+1) r^{-m+1} \\
-A_{3} m r^{m-1}-A_{4} m r^{-m-1}
\end{array}\right) \cos (m \theta) .
\end{gathered}
$$

Applying the boundary conditions Eq. B.2 to Eq. B.3 yields a linear system for $A_{i}$ and $B_{i}$, with solution

$$
B_{1}=\frac{1}{2\left(\beta^{2}+1-2 \nu_{S}\right)}\left[\overline{E_{S}} v_{0}\left(1-\nu_{S}\right)-P \beta^{2}\right]
$$




$$
\begin{aligned}
B_{2}= & \frac{-R^{2} \beta^{2}}{\beta^{2}+1-2 \nu_{S}}\left[\overline{E_{S}} v_{0}\left(1-\nu_{S}\right)+P\left(1-2 \nu_{S}\right)\right] \\
A_{1}= & \frac{(m-1) \beta^{2 m+2}+(\alpha-m+1) \beta^{2 m}+\beta^{2}}{2\left(-m^{2}+1\right) \beta^{2 m+2}+m^{2} \beta^{2 m+4}+\beta^{4 m+2} \alpha+\left(m^{2}-1+\alpha^{2}\right) \beta^{2 m}+\alpha \beta^{2}} G R^{-m} A \\
& +\frac{(m-1) \beta^{2 m+2}+(-m-\alpha+1) \beta^{2 m}-\beta^{2}}{2\left(-m^{2}+1\right) \beta^{2 m+2}+m^{2} \beta^{2 m+4}+\beta^{4 m+2} \alpha+\left(m^{2}-1+\alpha^{2}\right) \beta^{2 m}+\alpha \beta^{2}} G R^{-m} B, \\
A_{2}= & \frac{-(m+1) \beta^{2 m+2}+\beta^{4 m+2}+\beta^{2 m}(\alpha+m+1)}{2\left(-m^{2}+1\right) \beta^{2 m+2}+m^{2} \beta^{2 m+4}+\beta^{4 m+2} \alpha+\left(m^{2}-1+\alpha^{2}\right) \beta^{2 m}+\alpha \beta^{2}} G R^{m} A \\
& +\frac{(m+1) \beta^{2 m+2}+\beta^{4 m+2}+\beta^{2 m}(\alpha-m-1)}{2\left(-m^{2}+1\right) \beta^{2 m+2}+m^{2} \beta^{2 m+4}+\beta^{4 m+2} \alpha+\left(m^{2}-1+\alpha^{2}\right) \beta^{2 m}+\alpha \beta^{2}} G R^{m} B, \\
A_{3}= & -\frac{\beta^{2}\left((\alpha-m+1)(m+1) \beta^{2 m}+m^{2} \beta^{2 m+2}+\alpha+1+m\right)}{\left(2\left(-m^{2}+1\right) \beta^{2 m+2}+m^{2} \beta^{2 m+4}+\beta^{4 m+2} \alpha+\left(m^{2}-1+\alpha^{2}\right) \beta^{2 m}+\alpha \beta^{2}\right) m} G R^{-m+2} A \\
- & \frac{\beta^{2}\left(m^{2} \beta^{2 m+2}-1+(-m-\alpha+1)(m+1) \beta^{2 m}-m+\alpha\right)}{\left(2\left(-m^{2}+1\right) \beta^{2 m+2}+m^{2} \beta^{2 m+4}+\beta^{4 m+2} \alpha+\left(m^{2}-1+\alpha^{2}\right) \beta^{2 m}+\alpha \beta^{2}\right) m} G R^{-m+2} B, \\
A_{4}= & -\frac{\left(-m^{2} \beta^{2 m+2}+(m-1)(\alpha+m+1) \beta^{2 m}-(\alpha-m+1) \beta^{4 m}\right) \beta^{2}}{\left(2\left(-m^{2}+1\right) \beta^{2 m+2}+m^{2} \beta^{2 m+4}+\beta^{4 m+2} \alpha+\left(m^{2}-1+\alpha^{2}\right) \beta^{2 m}+\alpha \beta^{2}\right) m} G R^{m+2} A \\
& -\frac{\left(m^{2} \beta^{2 m+2}+(m-1)(\alpha-m-1) \beta^{2 m}-(-m-\alpha+1) \beta^{4 m}\right) \beta^{2}}{\left(2\left(-m^{2}+1\right) \beta^{2 m+2}+m^{2} \beta^{2 m+4}+\beta^{4 m+2} \alpha+\left(m^{2}-1+\alpha^{2}\right) \beta^{2 m}+\alpha \beta^{2}\right) m} G R^{m+2} B
\end{aligned}
$$

Substituting $A_{i}$ and $B_{i}$ into Eq. B.3 yields the stress at the interface $r=R$

$$
\begin{aligned}
& \sigma_{r r}(R, \theta)=K_{0} R v_{0}+K R \varepsilon A \sin (m \theta)-\gamma P, \\
& \sigma_{r \theta}(R, \theta)=\mathrm{M} R \varepsilon B \cos (m \theta),
\end{aligned}
$$

where

$$
\begin{aligned}
K_{0} & =\overline{E_{S}} \frac{1}{R} \frac{\left(1-\nu_{S}\right)\left(1-\beta^{2}\right)}{1-2 \nu_{S}+\beta^{2}} \\
K & =\overline{E_{S}} \frac{1}{R} \frac{\left(1-\nu_{S}\right)}{2}\left(S_{A}+S_{B} \frac{B}{A}\right), \\
\mathrm{M} & =\overline{E_{S}} \frac{1}{R} \frac{\left(1-\nu_{S}\right)}{2}\left(S_{B} \frac{A}{B}+T_{B}\right), \\
\gamma & =\frac{2 \beta^{2}\left(1-\nu_{S}\right)}{1-2 \nu_{S}+\beta^{2}}
\end{aligned}
$$

and

$$
\begin{aligned}
\psi S_{A}= & 2\left(-m^{2}\left(\beta^{4}+2+\alpha\right)+(3+\alpha)\left(m^{2}-1\right) \beta^{2}+2(\alpha+1)\right) \beta^{2 m} \\
& -\left(((\alpha+1) m+\alpha-1) \beta^{4 m}-(\alpha+1) m+\alpha-1\right) \beta^{2} \\
\psi S_{B}= & -2 m\left(-m^{2}\left(\beta^{4}+1\right)+2\left(m^{2}-1\right) \beta^{2}+1+\alpha\right) \beta^{2 m} \\
& +\left(((\alpha-1) m+\alpha+1) \beta^{4 m}+(\alpha-1) m-\alpha-1\right) \beta^{2}
\end{aligned}
$$




$$
\begin{gathered}
\psi T_{B}=2\left(-m^{2} \beta^{4}+(1-\alpha)\left(m^{2}-1\right) \beta^{2}+\alpha m^{2}\right) \beta^{2 m} \\
-\left(((\alpha+1) m+\alpha-1) \beta^{4 m}-(\alpha+1) m+\alpha-1\right) \beta^{2}, \\
\psi=\left(m^{2} \beta^{4}-\left(m^{2}-1\right)\left(2 \beta^{2}-1\right)+\alpha^{2}\right) \beta^{2 m}+\alpha \beta^{2}\left(1+\beta^{4 m}\right) .
\end{gathered}
$$

The dimensionless stress at the interface and the dimensionless stiffness parameters are obtained from Eq. B.5, by dividing it with $\overline{E_{F}}$,

$$
\begin{aligned}
\frac{\sigma}{\overline{E_{F}}} & =k_{0} v_{0}+k \varepsilon A \sin (m \theta)-\gamma p, \\
\frac{\tau}{\overline{E_{F}}} & =\mu \varepsilon B \sin (m \theta), \\
k_{0} & =\frac{K_{0} R}{\overline{E_{F}}}=\frac{\left(1-\nu_{S}\right)\left(1-\beta^{2}\right)}{1-2 \nu_{S}+\beta^{2}} \frac{1}{\xi}, \\
k & =\frac{K R}{\overline{E_{F}}}=\frac{\left(1-\nu_{S}\right)}{2}\left(S_{A}+S_{B} \frac{B}{A}\right) \frac{1}{\xi}, \\
\mu & =\frac{\mathrm{M} R}{\overline{E_{F}}}=\frac{\left(1-\nu_{S}\right)}{2}\left(S_{B} \frac{A}{B}+T_{B}\right) \frac{1}{\xi},
\end{aligned}
$$

as indicated in Eq. 10 .

We note that in the case of an inextensible wrinkling mode, $A / B=m$, the stiffness $K$ simplifies to $\widetilde{K}$ given by

$$
\begin{aligned}
\widetilde{K} & =\overline{E_{S}} \frac{1}{R} \frac{\left(1-\nu_{S}\right)}{2}\left(S_{A}+S_{B} \frac{1}{m}\right) \\
& =\overline{E_{S}} \frac{1}{m R} \frac{2\left(1-\nu_{S}\right)^{2}\left(\beta^{-2 m}-\beta^{2 m}+2 m\left(1-\beta^{-2}\right)\right)\left(m^{2}-1\right)}{\left(\beta^{-1}-\beta\right)^{2}\left(m^{2}-1\right)+\left(\beta^{-m}-\beta^{m}\right)^{2}\left(3-4 \nu_{S}\right)+\left(\frac{3-4 \nu_{S}}{\beta}+\beta\right)^{2}},
\end{aligned}
$$

which, for a substrate with no cavity, leads to the limiting case

$$
\lim _{\beta \rightarrow 0}(\widetilde{K})=\overline{E_{S}} \frac{1}{m R} \frac{2\left(1-\nu_{S}\right)^{2}\left(m^{2}-1\right)}{3-4 \nu_{S}},
$$

and, for an infinite plane substrate, yields

$$
\lim _{R \rightarrow \infty}\left(\lim _{\beta \rightarrow 0}(\widetilde{K})\right)=K_{\text {Plane }}=\overline{E_{S}} \frac{4\left(1-\nu_{S}\right)^{2}}{3-4 \nu_{S}} \frac{\pi}{\lambda}
$$

in agreement with Audoly and Boudaoud (2008a). 


\section{Appendix C. Terms of the linear stability analysis}

The terms $a_{i}, \widetilde{a_{i}}, b_{i}$ and $\widetilde{b_{i}}$ that appear in Eq. 16 are

$$
\begin{aligned}
& a_{1}=-4 \xi h\left(\left(m^{4}+\frac{3}{2}-\frac{11}{4} m^{2}\right) h^{2}-9-3 m^{2}\right) \frac{\gamma}{h+h^{3} / 12+k_{0}}, \\
& \widetilde{a_{1}}=h\left(\left(m^{2}-1\right)^{2} h^{2}+12\right) \xi+6\left(1-\nu_{S}\right) S_{A}, \\
& b_{1}=-4 \xi h\left(\frac{1}{4} h^{2} m^{3}+12 m\right) \frac{\gamma}{h+h^{3} / 12+k_{0}}, \\
& \widetilde{b_{1}}=-12 \xi h m+6\left(1-\nu_{S}\right) S_{B}, \\
& a_{2}=-\left(h^{3} m^{2}+48 h+12 k_{0}(1-\chi)\right) m \xi \frac{\gamma}{h+h^{3} / 12+k_{0}}+12 \gamma(1-\chi) m \xi, \\
& \widetilde{a_{2}}=-12 h m \xi+6\left(1-\nu_{S}\right) S_{B}, \\
& b_{2}=\left(36 h m^{2}+h^{3}+12 h+12 k_{0}(1-\chi)\right) \xi \frac{\gamma}{h+h^{3} / 12+k_{0}}-12 \gamma(1-\chi) \xi, \\
& \widetilde{b_{2}}=12 h m^{2} \xi+6\left(1-\nu_{S}\right) T_{B}
\end{aligned}
$$

where $\gamma, k_{0}, S_{A}, S_{B}$ and $T_{B}$ were given in Appendix B.

\section{Appendix D. Influence of the cavity size}

In Figs. D.8 and D.9 we plot the $h$ dependence of $\sigma_{c} / \sigma_{\text {Plane }}$ and $\lambda_{c} / \lambda_{\text {Plane }}$, for cavity sizes $\beta=0.2$ and $\beta=0.8$, respectively. These plots are qualitatively similar to those obtained for $\beta=0.5$ in Figs. 6 and 7 . and discussed in $\$ 5.4$ of the main text.

\section{References}

Allen, H.G., 1969. Analysis and Design of Structural Sandwich Panels. Pergamon, New York.

Atanackovic, T.M., 1998. Buckling of a compressible elastic ring. Acta Mechanica 127, 121-134.

Audoly, B., Boudaoud, A., 2008a. Buckling of a thin film bound to a compliant substrate (part I). Formulation, linear stability of cylindrical patterns, secondary bifurcations. Journal of the Mechanics and Physics of Solids 56, 2401-2421.

Audoly, B., Boudaoud, A., 2008b. Buckling of a thin film bound to a compliant substrate (part II). A global scenario for the formation of herringbone pattern. Journal of the Mechanics and Physics of Solids 56, 2422-2443.

Audoly, B., Boudaoud, A., 2008c. Buckling of a thin film bound to a compliant substrate (part III). Herringbone solutions at large buckling parameter. Journal of the Mechanics and Physics of Solids 56, 2444-2458.

Barber, J.R., 2002. Elasticity: 2nd Edition. Kluwer Academic Publishers.

Ben Amar, M., Goriely, A., 2005. Growth and instability in elastic tissues. Journal of the Mechanics and Physics of Solids 53, $2284-2319$.

Biot, M., 1965. Mechanics of Incremental Deformation. Wiley, New York.

Bowden, N., Brittain, S., Evans, A.G., Hutchinson, J.W., Whitesides, G.M., 1998. Spontaneous formation of ordered structures in thin films of metals supported on an elastomeric polymer. Nature 393, 146-149.

Breid, D., Crosby, A.J., 2009. Surface wrinkling behavior of finite circular plates. Soft Matter 5, 425-431.

Breid, D., Crosby, A.J., 2013. Curvature-controlled wrinkle morphologies. Soft Matter 9, 3624-3630.

Brush, D.O., Almroth, B.O., 1975. Buckling of Bars, Plates and Shells. McGraw-Hill, New York.

Budday, S., Steinmann, P., Kuhl, E., 2014. The role or mechanics during brain development. Journal of the Mechanics and Physics of Solids http://dx.doi.org/10.1016/j.jmps.2014.07.010.

Cai, S., Bertoldi, K., Wang, H., Suo, Z., 2010. Osmotic collapse of a void in an elastomer: breathing, buckling and creasing. Soft Matter 6, 5770-5777.

Cai, S., Breid, D., Crosby, A.J., Suo, Z., Hutchinson, J.W., 2011. Periodic patterns and energy states of buckled films on compliant substrates. Journal of the Mechanics and Physics of Solids 59, 1094-1114.

Cao, Y.P., Li, B., Feng, X.Q., 2012. Surface wrinkling and folding of core-shell soft cylinders. Soft Matter 8, 556.

Carrier, G.F., 1947. On the buckling of elastic rings. Journal of Mathematics and Physics 26, 94-103.

Chan, E.P., Crosby, A.J., 2006a. Fabricating microlens arrays by surface wrinkling. Advanced Materials 18(24), 3238-3242.

Chan, E.P., Crosby, A.J., 2006b. Spontaneous formation of stable aligned wrinkling patterns. Soft Matter 2(4), 324-328. 
Chen, X., Hutchinson, J.W., 2004. Herringbone buckling patterns of compressed thin films on compliant substrates. Journal of Applied Mechanics 71(5), 597-603.

Chen, X., Yin, J., 2010. Buckling patterns of thin films on curved compliant substrates with applications to morphogenesis and three-dimensional micro-fabrication. Soft Matter 22(6), 5667-5680.

Cheng, P., 1996. Weight optimization of cylindrical shells with cellular cores. Master of Science manuscript.

Combescure, A., 1981. Calcul analytique de la déformation d'un anneau avec défauts. DEMT/SMTS/BAMS/81-142 F.E. : 5423-05-000-09.

Efimenko, K., Rackaitis, M., Manias, E., Vaziri, A., Mahadevan, L., Genzer, J., 2005. Nested self-similar wrinkling patterns in skins. Nature Materials 4, 293-297.

Genzer, J., Groenewold, J., 2006. Soft matter with hard skin: From skin wrinkles to templating and material characterization. Soft Matter 2, 310-323.

Huang, Z., Hong, W., Suo, Z., 2005. Nonlinear analyses of wrinkles in a film bonded to a compliant substrate. Journal of the Mechanics and Physics of Solids 53, 2101-2118.

Huck, W.T.S., Bowden, N., Onck, P., Pardoen, T., Hutchinson, J.W., Whitesides, G.M., 2000. Ordering of spontaneously formed buckles on planar surfaces. Langmuir 16, 3497-3501.

Huddleston, P.J., Lan, L., 1993. Information from fold shapes. Journal of Structural Geology 15, $253-264$.

Kachman, D., 1959. Test report on buckling of propellant cylinders under compressive loads. Space Technology Labs, Inc., GM-59-7520.6-24.

Kim, D.H., Lu, N., Ma, R., Kim, Y.S., Kim, R.H., Shuodao, W., Wu, J., Won, S.M., Kim, T.I., Chowdhury, R., Ying, M., Xu, L., Li, M., Chung, H.J., Keum, H., McCormick, M., Liu, P., Zhang, Y.W., Omenetto, F.G., Huang, Y., Coleman, T., Rogers, J.A., 2011. Epidermal electronics. Science 333, 838.

Kim, J.B., Kim, P., Pégard, N.C., Oh, S.J., Kagan, C.R., Fleischer, J.W., Stone, H.A., Loo, Y.L., 2012. Wrinkles and deep folds as photonic structures in photovoltaics. Nature Photonics 6, 327-332.

Kundu, S., Davis, C., Long, T., Sharma, R., Crosby, A., 2011. Adhesion of nonplanar wrinkled surfaces. Journal of Polymer Science Part B: Polymer Physics 49, 179-185.

Landau, L.D., Lifshitz, E.M., 1959. Theory of Elasticity. Pergamon, London.

Lévy, M.M., 1884. Mémoire sur un nouveau cas intégrable du problème de l'élastique et de l'une de ses applications. Journal de Mathématiques Pures et Appliquées 3X, 5-42.

Li, B., Cao, Y.P., Feng, X.Q., 2011a. Growth and surface folding of esophageal mucosa: a biomechanical model. Journal of Biomechanics 44, 182-188.

Li, B., Cao, Y.P., Feng, X.Q., Gao, H., 2011b. Surface wrinkling of mucosa induced by volumetric growth : Theory, simulation and experiment. Journal of the Mechanics and Physics of Solids 59, 758-774.

Li, B., Cao, Y.P., Feng, X.Q., Gao, H., 2012. Mechanics of morphological instabilities and surface wrinkling in soft materials: a review. Soft Matter 8, 5728 .

Li, B., F, J., Cao, Y.P., Feng, X.Q., Gao, H., 2011c. Surface wrinkling patterns on a core-shell soft sphere. Physical Review Letters 106, 234301.

Lourie, O., Cox, D.M., Wagner, H.D., 1998. Buckling and collapse of embedded carbon nanotubes. Physical Review Letters $81,1638-1641$.

Mei, H., Landis, C., Huang, R., 2011. Concomitant wrinkling and buckle-delamination of elastic thin films on compliant substrates. Mechanics of Materials 43, 627-642.

Mei, Y., Kiravittaya, S., Harazim, S., Schmidt, O.G., 2010. Principles and applications of micro and nanoscale wrinkles. Materials Science and Engineering R 70, 209-224.

Michel, J., Lopez-Pamies, O., Ponte Castañeda, P., Triantafyllidis, N., 2007. Microscopic and macroscopic instabilities in finitely strained porous elastomers. Journal of the Mechanics and Physics of Solids 55, 900-938.

Michell, J.H., 1899. On the direct determination of stress in an elastic solid, with application to the theory of plates. Proceedings of the London Mathematical Society s1-31(1), 100-124.

Price, N.J., Cosgrove, J.W., 1990. Analysis of Geological Structures. Cambridge University Press, Cambridge.

Seide, P., 1962. The stability under axial compression and lateral pressure of circular-cylindrical shells with a soft elastic core. Journal of the Aerospace Sciences 29(7), 851-862

Seide, P., Weingarten, V.I., 1961. Buckling of circular rings and long cylinders enclosing an elastic material under uniform external pressure. American Rocket Society Journal 32(5), 680-687.

Terwagne, D., Brojan, M., Reis, P., 2014. Smart morphable surfaces for aerodynamic drag control. Advanced Materials , In press.

Timoshenko, S.P., Gere, J.M., 1961. Theory of Elastic Stability. McGraw-Hill, New York.

Yin, J., Chen, X., Sheinman, I., 2009. Anisotropic buckling patterns in spheroidal film/substrate systems and their implications in some natural and biological systems. Journal of the Mechanics and Physics of Solids 57, 1470-1484.

Yin, J., Han, X., Cao, Y., Lu, C., 2014. Surface wrinkling on polydimethylsiloxane microspheres via wet surface chemical oxidation. Scientific Reports 4, 5710

Yin, J., Yagüe, J.L., Eggenspieler, D., Gleason, K.K., Boyce, M.C., 2012. Deterministic order in surface micro-topologies through sequential wrinkling. Advanced materials 24, 5441-5446.

Yoo, P.J., Suh, K.Y., Park, S.Y., Lee, H.H., 2002. Physical self-assembly of microstructures by anisotropic buckling. Advanced materials 14, 1383-1387.

Yu, C., Jiang, H., 2010. Forming wrinkled stiff films on polymeric substrates at room temperature for strechable interconnects applications. Thin Solid Films 519, 818-822. 


\section{Figures}

a)

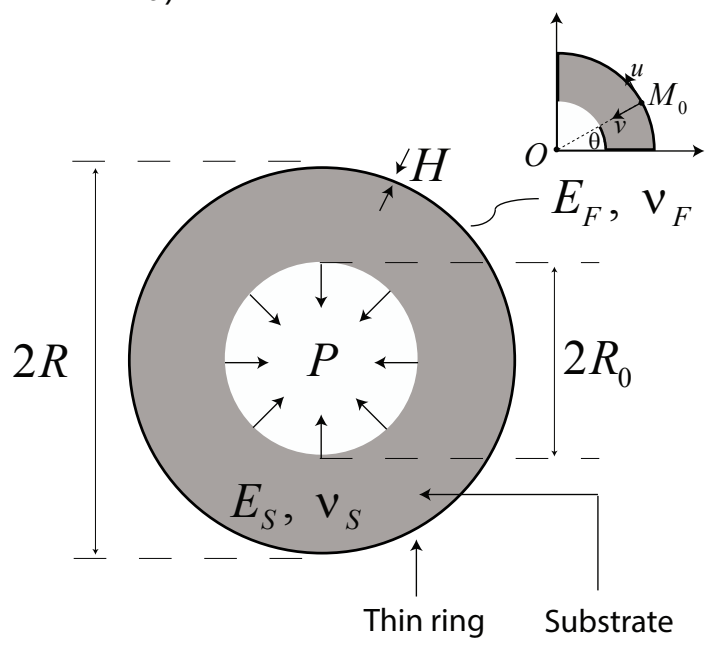

b)

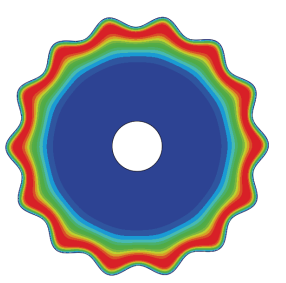

d)

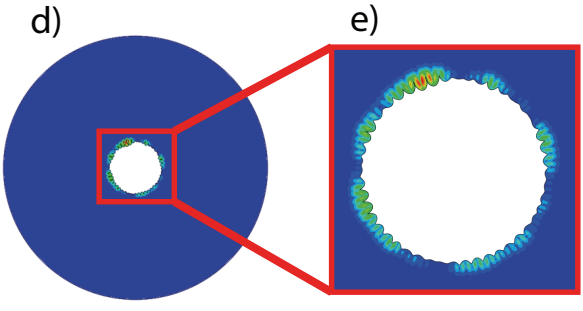

C)

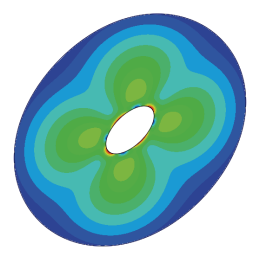

Normalized

Maximum

Principal

Strain

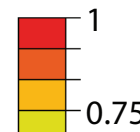

0.5

0.25

Figure 1: (a) Schematic diagram of our system: a ring is bound to a curved substrate which contains a circular cavity. The system is loaded by applying a pressure differential between the inside of the cavity and the outside of the ring. (b-d) Representative examples of the three possible instability modes of a ring on a curved substrate which contains a cavity that is depressurized. (b) Wrinkling mode $\left(h=10^{-2}\right.$ and $\left.\xi=10^{3}\right)$, (c) global buckling mode $\left(h=10^{-2}\right.$ and $\left.\xi=10^{6}\right)$ and $(\mathrm{d})$ Biot mode $\left(h=10^{-2}\right.$ and $\left.\xi=10^{2}\right)$. (e) is a zoom in of (d) that exhibits the deformation of the surface of the inner cavity in the Biot mode. The adjacent colorbar applies to pictures b-e) and refers to the maximum principal component of the strain tensor of the mode, which has been normalized by the maximum value of each configuration. 


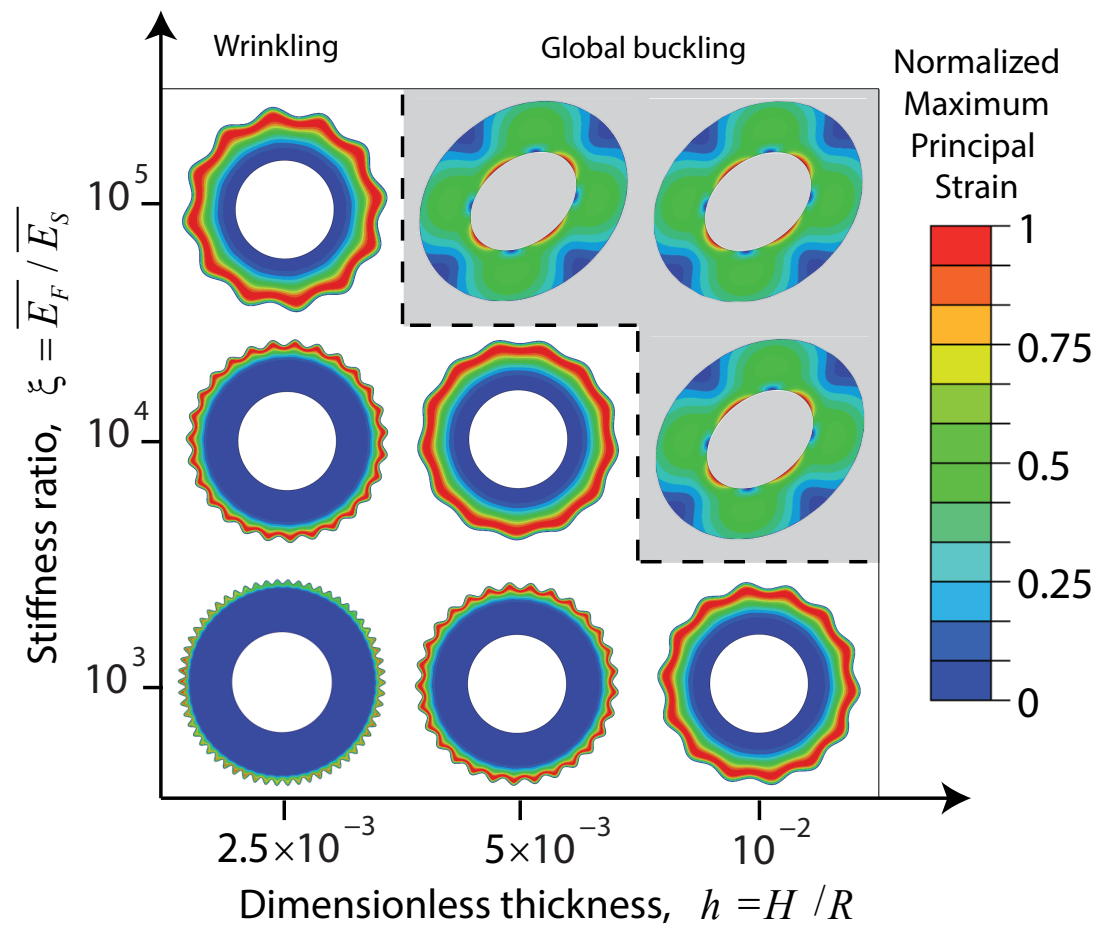

Figure 2: Schematic phase diagram of the instability modes of the system, obtained for a critical value of the pressure differential: i) wrinkling of the ring or ii) global buckling of the structure (shaded regions). The primary parameters that govern this transition are the stiffness and thickness ratios: $\xi=\overline{E_{F}} / \overline{E_{S}}$ and $h=H / R$, respectively. The depicted examples from FEM simulations are for an incompressible film and substrate, $\nu_{F}=\nu_{S}=0.5$. They were obtained for $R=100$ units of length and $E_{S}=1$ units of pressure, while varying $H$ and $E_{F}$. The colorbar refers to the maximum principal component of the strain tensor of the mode, which has been normalized by the maximum value of each configuration. 
a)

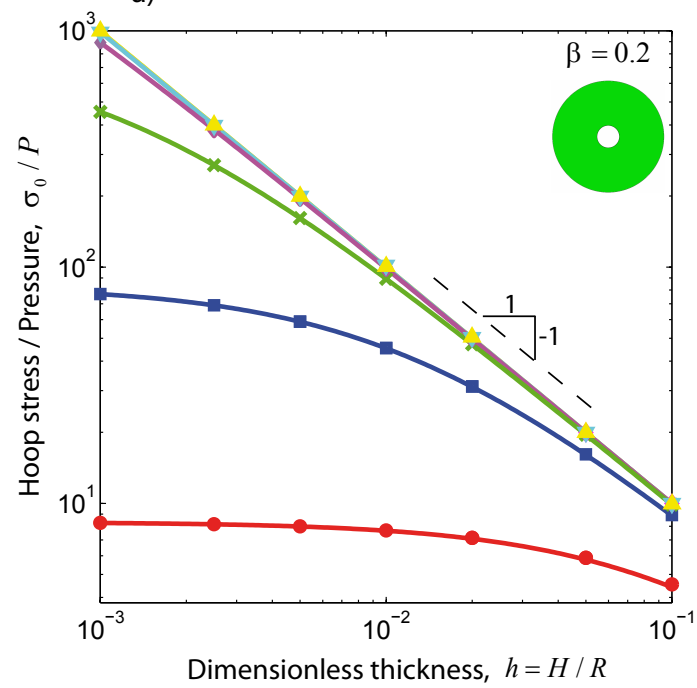

c)

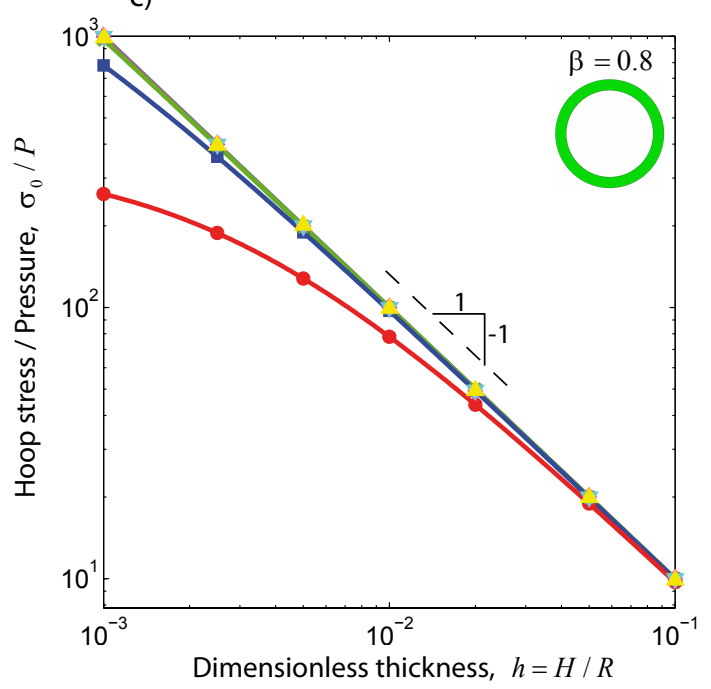

b)
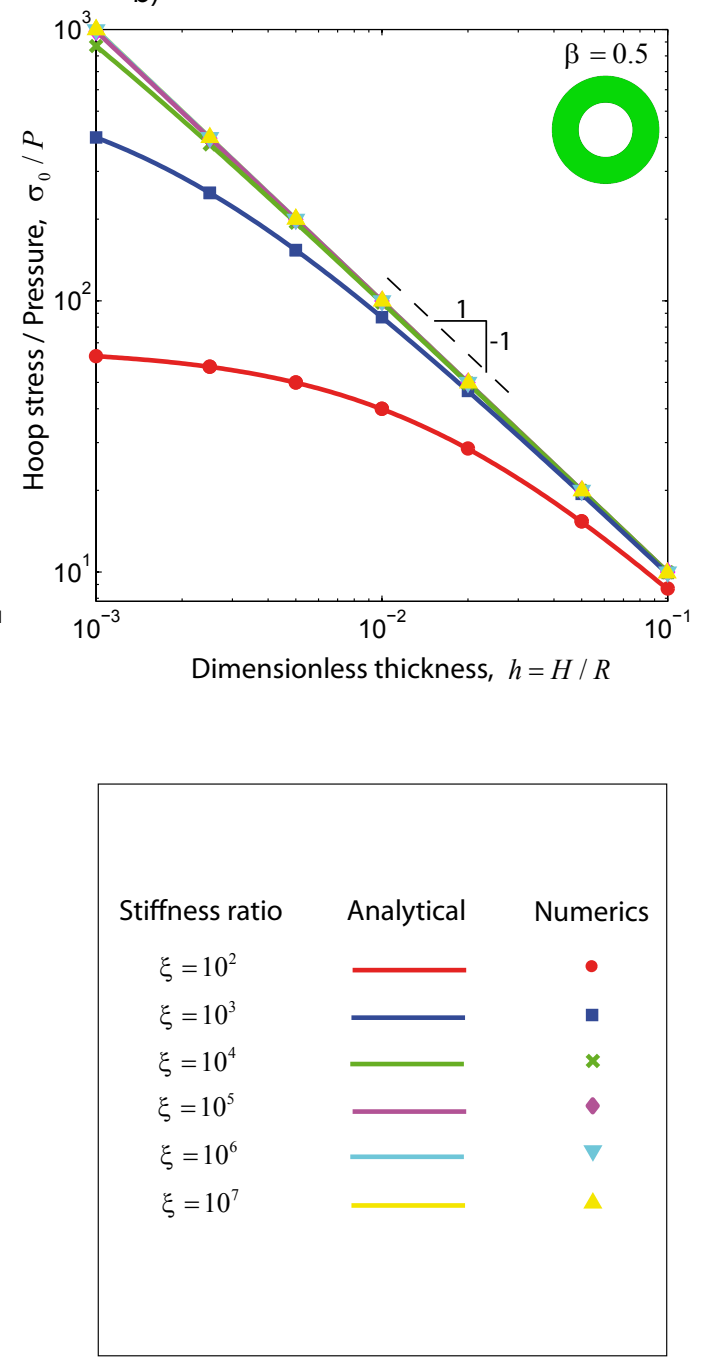

Figure 3: Pre-instability hoop stress, $\sigma_{0}$, in the ring, normalized by the pressure, $P$, as a function of the dimensionless ring thickness, $h=H / R$. Cavity sizes are: (a) $\beta=0.2$, (b) $\beta=0.5$ and (c) $\beta=0.8$. The Poisson's ratio of the substrate is $\nu_{S}=0.5$. Analytical predictions are given by Eq. $\sqrt{12}$ ) as solid lines and FEM results are plotted as data points. The legend (bottom right) is common to all three plots. 
a)

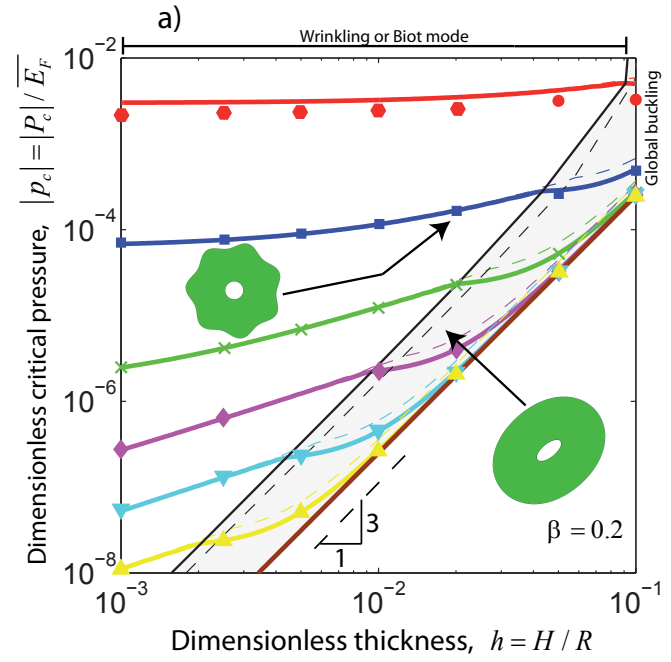

C)

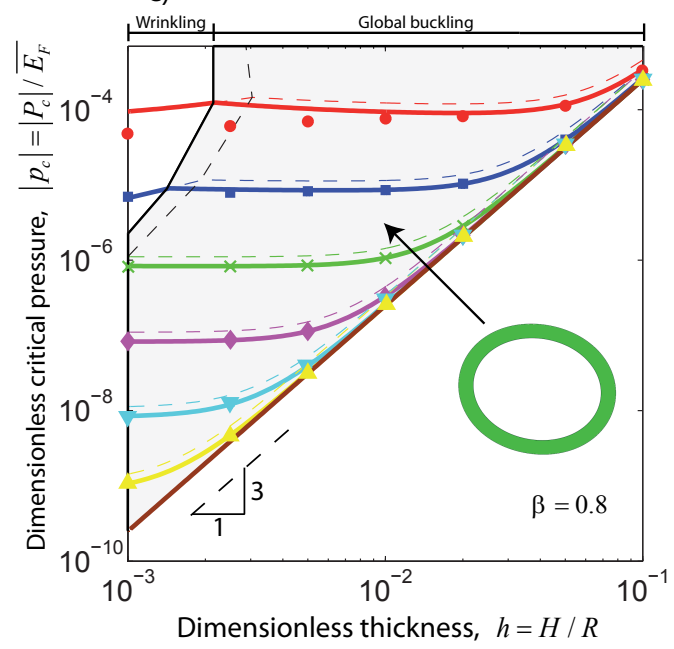

b)
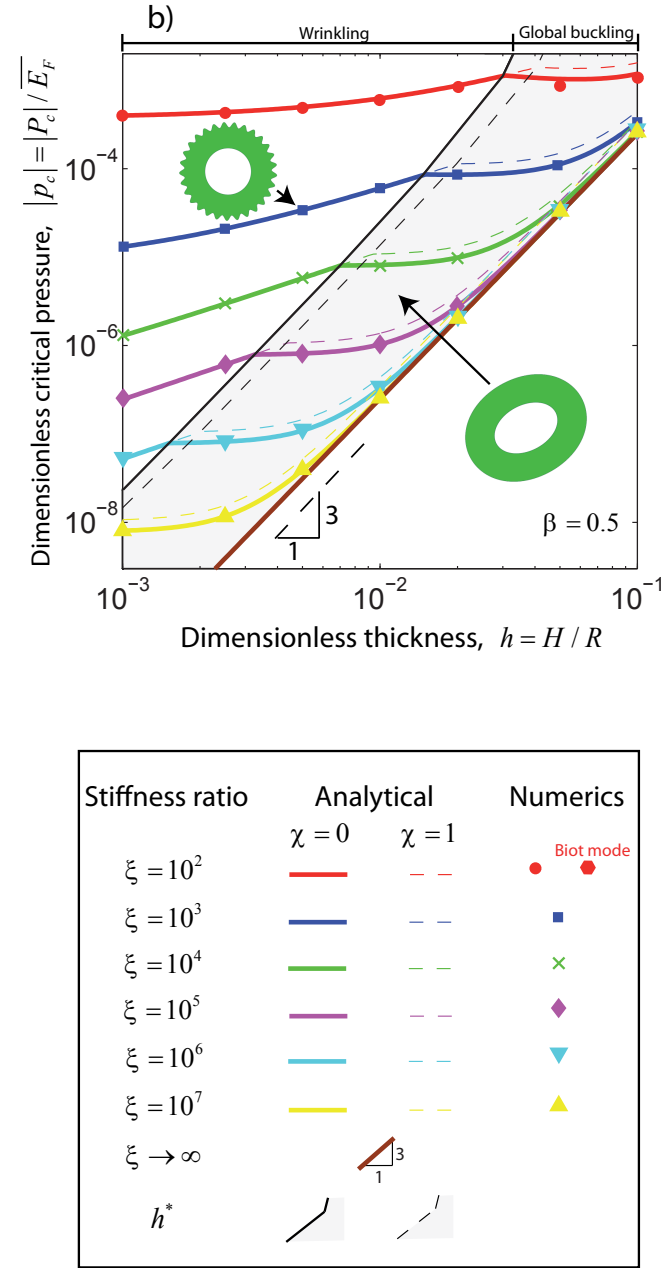

Figure 4: Dimensionless critical pressure $\left|p_{c}\right|=\left|P_{c}\right| / \overline{E_{F}}$ versus the dimensionless ring thickness $h=H / R$, for several stiffness ratios $\xi=\overline{E_{F}} / \overline{E_{S}}$ and cavity sizes $\beta$ : (a) $\beta=0.2$, (b) $\beta=0.5$ and (c) $\beta=0.8$. Theory (lines) is given by Eq. 17 . The Poisson's ratio of the substrate is $\nu_{S}=0.5$. The legend (bottom right) is common to all three plots. 
a)
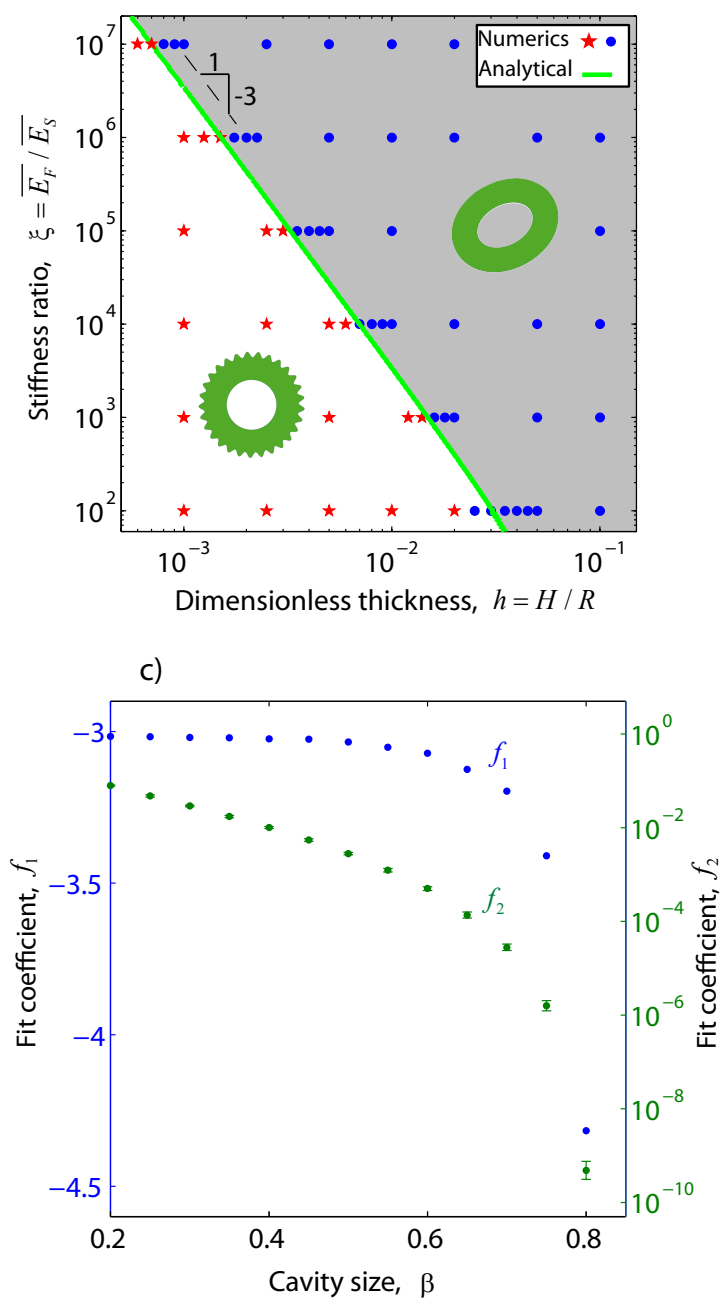

b)

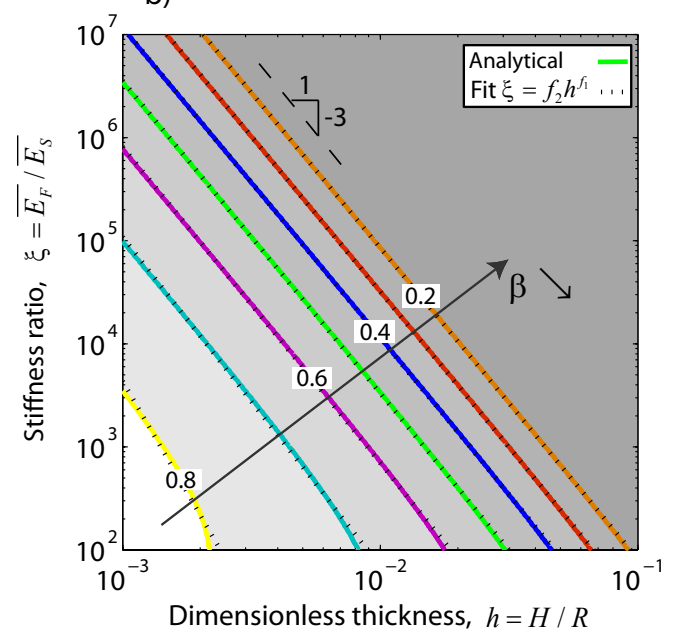

d)

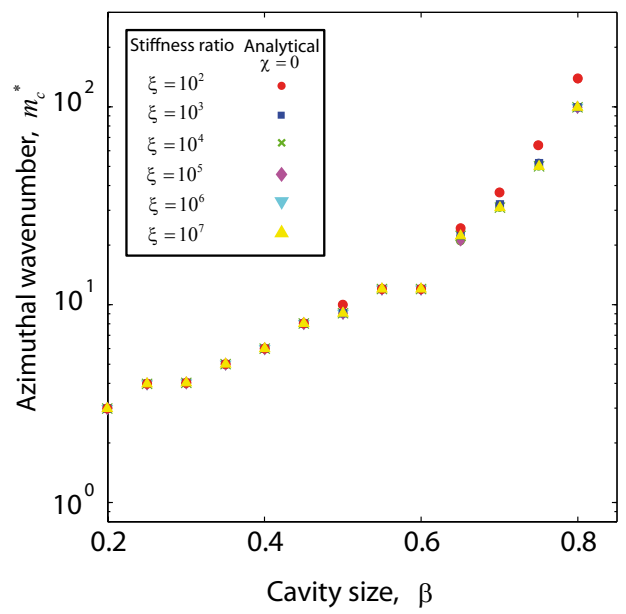

Figure 5: (a) Phase diagram in the $(\xi, h)$ parameter space, showing the transition boundary, $h^{*}$, from wrinkling to global buckling, for $\beta=0.5$. (b) Phase diagram for $\beta=0.2$ to $\beta=0.8$. Solid lines are analytical predictions and dotted lines correspond to the numerical fit $\xi=f_{2} h^{f_{1}}$. (c) Fitting coefficients, $f_{1}$ and $f_{2}$, as functions of $\beta$. (d) Azimuthal wavenumber of the wrinkling mode for $h=h^{*}$, as a function of $\beta$. The Poisson's ratio of the substrate is $\nu_{S}=0.5$. 

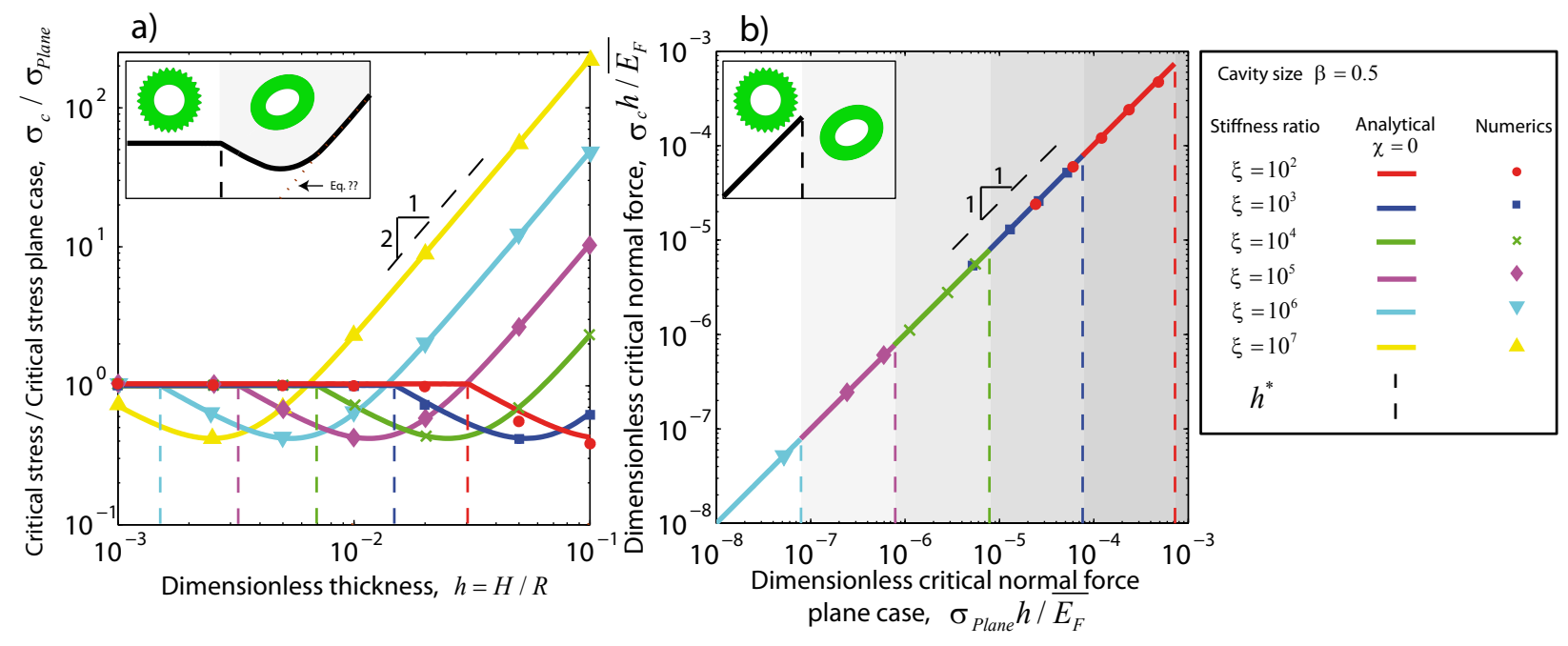

Figure 6: (a) Critical stress $\sigma_{c}$ normalized by its planar substrate counterpart, $\sigma_{\text {Plane }}$, as a function of the dimensionless ring thickness $h=H / R$. (b) Dimensionless critical normal force versus planar substrate counterpart. Analytical predictions (solid lines) are given by Eq. 21a and FEM results are shown as data points. Insets are sketches of the wrinkling and global buckling modes. The cavity size is $\beta=0.5$ and the Poisson's ratio of the substrate is $\nu_{S}=0.5$. The legend is common to both plots. 

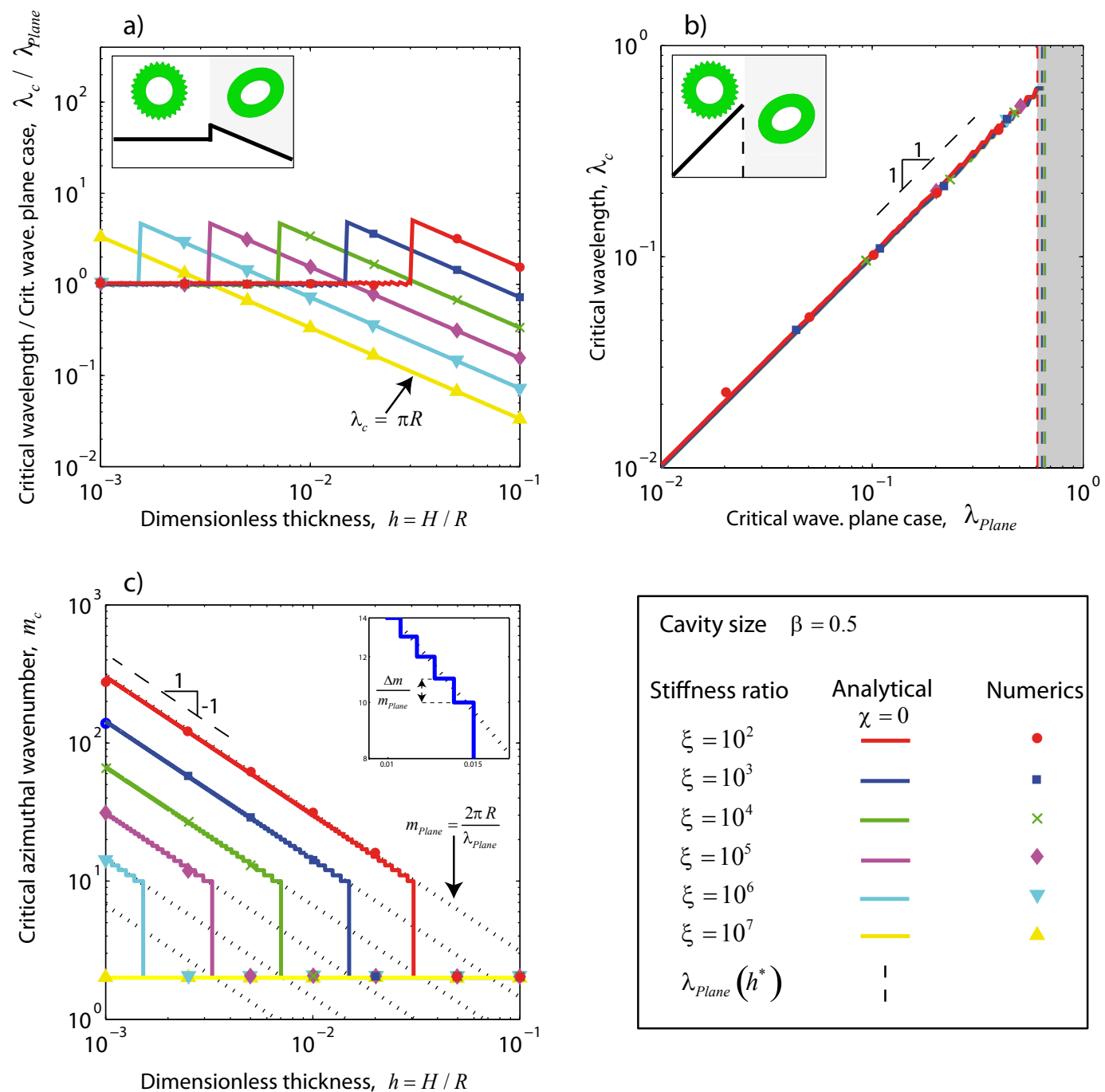

\begin{tabular}{|ccc|}
\hline Cavity size & $\beta=0.5$ & \\
Stiffness ratio & Analytical & Numerics \\
$\xi=10^{2}$ & $\underline{\chi=0}$ & \\
$\xi=10^{3}$ & - & \\
$\xi=10^{4}$ & - & $\times$ \\
$\xi=10^{5}$ & - & \\
$\xi=10^{6}$ & - & \\
$\xi=10^{7}$ & - & \\
$\lambda_{\text {Plane }}\left(h^{*}\right)$ & I & \\
\hline
\end{tabular}

Figure 7: (a) Critical wavelength, $\lambda_{c}$, normalized by its planar substrate counterpart, $\lambda_{\text {Plane }}$, as a function of the dimensionless ring thickness $h=H / R$. Analytical predictions (solid lines) are given by Eq. 21b. (b) Zoom of the wrinkling domain by plotting $\lambda_{c}$ versus $\lambda_{\text {Plane. }}$ (c) Azimuthal wavenumber $m_{c}$ of the wrinkling mode as a function of $h$. Analytical predictions (solid lines) are given by Eq. (17). Dotted lines show $m_{c}$ for the planar substrate case. Insets on (a) and (b) are sketches of the wrinkling and global buckling modes. Inset on (c) is a zoom in showing the difference in $m_{c}$ for the curved and planar substrates. The cavity size is $\beta=0.5$ and the Poisson's ratio of the substrate is $\nu_{S}=0.5$. FEM results shown as data points. The legend (bottom right) is common to all three plots. 

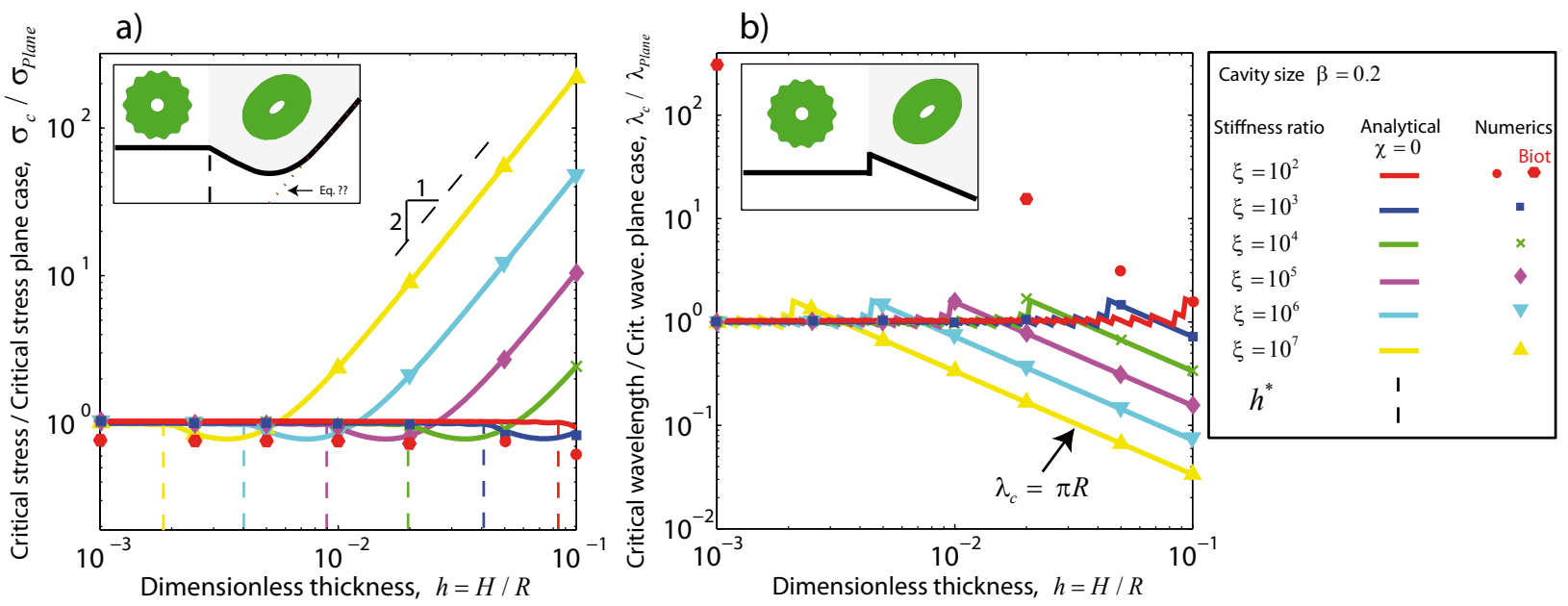

Figure D.8: (a) Critical stress $\sigma_{c}$ normalized by its planar substrate counterpart $\sigma_{\text {Plane }}$, as a function of the dimensionless ring thickness $h=H / R$. Analytical prediction is given by Eq. 21a. (b) Critical wavelength $\lambda_{c}$ normalized by its planar substrate counterpart $\lambda_{\text {Plane }}$, as a function of $h$. Analytical prediction is given by Eq. (21b). Insets are sketches of the wrinkling and global buckling modes. The cavity size is $\beta=0.2$ and the Poisson's ratio of the substrate is $\nu_{S}=0.5$. The legend is common to both plots.
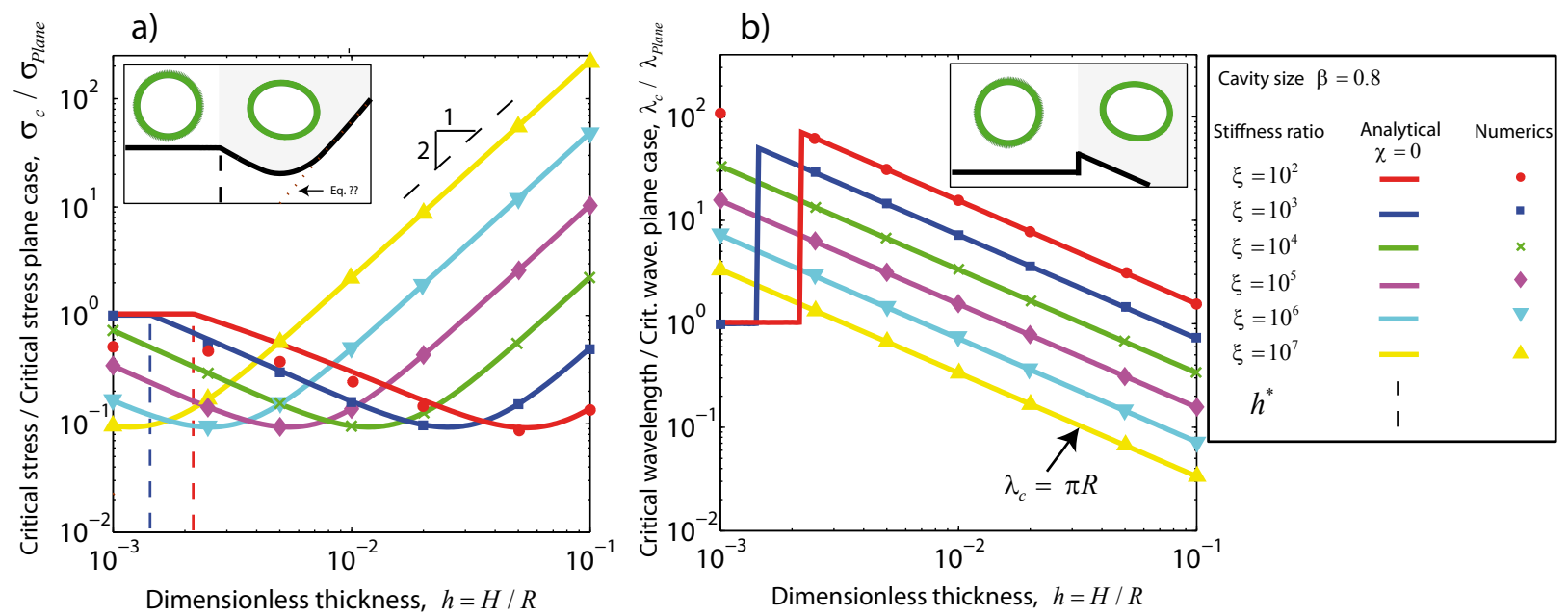

Figure D.9: (a) Critical stress $\sigma_{c}$ normalized by its plane substrate counterpart $\sigma_{\text {Plane }}$, as a function of the dimensionless ring thickness $h=H / R$. Analytical prediction is given by Eq. 21a. (b) Critical wavelength $\lambda_{c}$ normalized by its plane substrate counterpart $\lambda_{\text {Plane }}$, as a function of $h$. Analytical prediction is given by Eq. 21b). Insets are sketches of the wrinkling and global buckling modes. The cavity size is $\beta=0.8$ and the Poisson's ratio of the substrate is $\nu_{S}=0.5$. The legend is common to both plots. 